\title{
STAGGERED EXPLICIT-IMPLICIT TIME-DISCRETIZATION FOR ELASTODYNAMICS WITH DISSIPATIVE INTERNAL VARIABLES
}

\author{
Tomáš RoubíčEK ${ }^{1,2, *}$ And Chrysoula TsogkA ${ }^{3,4}$
}

\begin{abstract}
An extension of the two-step staggered time discretization of linear elastodynamics in stress-velocity form to systems involving internal variables subjected to a possibly non-linear dissipative evolution is proposed. The original scheme is thus enhanced by another step for the internal variables which, in general, is implicit, although even this step might be explicit if no spatial gradients of the internal variables are involved. Using an abstract Banach-space formulation, a priori estimates and convergence are proved under a CFL condition. The developed three-step scheme finds applications in various problems of continuum mechanics at small strain. Here, we consider in particular plasticity, viscoelasticity (creep), diffusion in poroelastic media, and damage.
\end{abstract}

Mathematics Subject Classification. 65M12, 65P10, 65Z05, 74C10, 74F10, 74H15, 74R20, 74S05, 76S05.

Received October 16, 2019. Accepted June 5, 2020.

\section{INTRODUCTION}

In computational mechanics one can distinguish two main classes of time-dependent problems, quasistatic and dynamic. Focusing on the latter one, one can further distinguish two other cases: (i) Low-frequency regimes, which are typically related with vibrations of structures and where the energy is not dominantly transmitted through space. In this case implicit time-discretization is relatively efficient, even though large systems of algebraic equations are to be solved at each time step. (ii) High-frequency regimes which arise typically within wave propagation and for which only explicit time-discretizations are reasonably efficient, in particular in threedimensions. These explicit methods can essentially be used in hyperbolic problems, as mere elastodynamics (treated here) or the elasto-magnetic Maxwell system or some conservation laws. Yet, many applications need to combine the convervative hyperbolic problems with various dissipative processes of a parabolic character, involving typically some internal variables. For parabolic problems, however, explicit methods are known to be problematic due to severe time-step restrictions. Therefore, we propose and analyse an explicit/implicit scheme,

Keywords and phrases. Elastodynamics, explicit discretization, fractional steps, mixed finite-element method, plasticity, creep, poro-elasticity, damage.

1 Mathematical Institute, Math.-Phys. Faculty, Charles University, Sokolovská 83, CZ-186 75 Praha 8, Czech Republic.

2 Institute of Thermomechanics of the Czech Acad. Sci., Dolejškova 5, CZ-182 08 Praha 8, Czech Republic.

3 Department of Applied Mathematics, University of California, Merced, 5200 North Lake road, Merced, CA 95343, USA.

4 Institute of Applied and Computational Mathematics, Foundation for Research and Technology - Hellas, Nikolaou Plastira 100, Vassilika Vouton, GR-700 13 Heraklion, Crete, Greece.

${ }^{*}$ Corresponding author: tomas.roubicek@mff.cuni.cz; roubicek@karlin.mff.cuni.cz 
using the fractional-step (also called staggered) technique. In fact, the proposed method becomes completely explicit in the absence of gradients of internal variables, as it can be in plasticicity or viscoelasticity as in Section 5.1, or an interfacial variant of damage models (so-called delamination) as in [43].

Our starting point is the linear elastodynamic problem: Find the displacement $u:[0, T] \times \Omega \rightarrow \mathbb{R}^{d}$ satisfying

$$
\begin{array}{ll}
\varrho \ddot{u}-\operatorname{div} \mathbb{C} e(u)=f & \text { on } \Omega \text { for } t \in(0, T], \\
{[\mathbb{C} e(u)] \boldsymbol{n}+\mathbb{B} u=g} & \text { on } \Gamma \text { for } t \in(0, T], \\
\left.u\right|_{t=0}=u_{0},\left.\quad \dot{u}\right|_{t=0}=v_{0} & \text { on } \Omega,
\end{array}
$$

for $T>0$ a fixed time horizon. Here $\Omega \subset \mathbb{R}^{d}$ is a bounded Lipschitz domain, $d=2$ or $3, \Gamma$ is its boundary, and $\boldsymbol{n}$ the unit outward normal. The dot-notation stands for the time derivative. In (1.1), $\varrho>0$ denotes the mass density, $\mathbb{C}$ is the elasticity tensor which is symmetric and positive definite, $e(u)$ denotes the small-strain tensor defined as $e(u)=\frac{1}{2}(\nabla u)^{\top}+\frac{1}{2} \nabla u$, and $\mathbb{B}$ is a symmetric positive semidefinite 2nd-order tensor determining the elastic support on the boundary. The terms appearing in the second member of (1.1) are, the bulk force $f$ and the surface loading $g$. In (1.1c), $u_{0}$ denotes the initial displacement and $v_{0}$ the initial velocity. For a more compact notation, we write the initial-boundary-value problem (1.1) in the following abstract form

$$
\mathcal{T}^{\prime} \ddot{u}+\mathcal{W}^{\prime} u=\mathcal{F}_{u}^{\prime}(t) \quad \text { for } t \in(0, T],\left.\quad u\right|_{t=0}=u_{0},\left.\quad \dot{u}\right|_{t=0}=v_{0} .
$$

Here $\mathcal{T}$ is the kinetic energy, $\mathcal{W}$ is the stored energy, and $\mathcal{F}$ is the external force, while $(\cdot)^{\prime}$ denotes the Gâteaux derivative. In the context of (1.1), we have

$$
\mathcal{T}(\dot{u})=\int_{\Omega} \frac{1}{2} \varrho|\dot{u}|^{2} \mathrm{~d} x, \quad \mathcal{W}(u)=\int_{\Omega} \frac{1}{2} \mathbb{C} e(u): e(u) \mathrm{d} x+\int_{\Gamma} \frac{1}{2} \mathbb{B} u \cdot u \mathrm{~d} S
$$

and

$$
\mathcal{F}(t, u)=\int_{\Omega} f(t) \cdot u \mathrm{~d} x+\int_{\Gamma} g(t) \cdot u \mathrm{~d} S
$$

Thus $\mathcal{F}_{u}^{\prime}(t)$ is a linear functional, let us denote it shortly by $F(t)$.

For high-frequency wave propagation problems, implicit time discretizations are computationally expensive, especially in the three dimensional setting. Therefore, we focus our attention on explicit methods. The simplest explicit scheme is the following second-order finite difference scheme

$$
\mathcal{T}^{\prime} \frac{u_{\tau h}^{k+1}-2 u_{\tau h}^{k}+u_{\tau h}^{k-1}}{\tau^{2}}+\mathcal{W}_{h}^{\prime} u_{\tau h}^{k}=F_{h}(k \tau)
$$

Here $\tau>0$ denotes the time step, and $\mathcal{W}_{h}$ (resp. $F_{h}$ ) denote some discrete approximations of the respective continuous functionals obtained by a suitable finite-element method (FEM) with mesh size $h>0$. For simplicity, we assume the mass density constant (or at least piecewise constant in space) so that the kinetic energy $\mathcal{T}$ does not need any numerical approximation. In particular, a numerical approximation leading to a diagonal the mass matrix $\mathcal{T}^{\prime}$ in (1.3), typically referred to as mass lumping, is an important ingredient so as to obtain efficient explicit methods. Here we will consider that $u$ is discretized in an element-wise constant way so that $\mathcal{T}^{\prime}$ leads to a diagonal matrix even without any approximation. Multiplying (1.3) by $\frac{u_{\tau h}^{k+1}-u_{\tau h}^{k}}{\tau}$ and using the scheme, it is easy to show that the energy preserved is

$$
\frac{1}{2}\left\langle\mathcal{T}^{\prime} \frac{u_{\tau h}^{k+1}-u_{\tau h}^{k}}{\tau}, \frac{u_{\tau h}^{k+1}-u_{\tau h}^{k}}{\tau}\right\rangle+\frac{1}{2}\left\langle\mathcal{W}_{h}^{\prime} u_{\tau h}^{k+1}, u_{\tau h}^{k}\right\rangle .
$$

This is an energy, i.e. a positive quantity under the following Courant-Fridrichs-Lewy (CFL) condition [20]

$$
\left\langle\mathcal{T}^{\prime} u_{h}, u_{h}\right\rangle \geq \frac{\tau^{2}}{4}\left\langle\mathcal{W}_{h}^{\prime} u_{h}, u_{h}\right\rangle
$$


for any $u_{h}$ from the respective finite-dimensional subspace. The CFL typically bounds the time discretization step $\tau=\mathscr{O}\left(h_{\min }\right)$ with $h_{\text {min }}$ the smallest element size on a FEM discretization. This method has frequently been used and analysed from various aspects, including comparison with implicit time discretizations, cf. e.g. $[33,34]$. However, the form of the discrete stored energy $\frac{1}{2}\left\langle\mathcal{W}_{h}^{\prime} u_{\tau h}^{k+1}, u_{\tau h}^{k}\right\rangle$ makes this discretization less suitable for the problem that we wish to consider in this paper where the stored energy is enhanced by some internal variables and (possibly) nonlinear processes on them.

Therefore, we use another explicit discretization scheme, the so-called leap-frog scheme. To this end, we first write the velocity/stress formulation of (1.1a). Introducing the velocity, $v=\dot{u}$ and the stress tensor $\sigma:=\mathbb{C} e(u)$, we get

$$
\begin{array}{ll}
\varrho \dot{v}-\operatorname{div} \sigma=f \quad \text { and } \quad \dot{\sigma}=\mathbb{C} e(v) & \text { on } \Omega \text { for } t \in(0, T], \\
\dot{\sigma} \boldsymbol{n}+\mathbb{B} v=\dot{g} & \text { on } \Gamma \text { for } t \in(0, T], \\
\left.v\right|_{t=0}=v_{0},\left.\quad \sigma\right|_{t=0}=\sigma_{0}:=\mathbb{C} e\left(u_{0}\right) & \text { on } \Omega .
\end{array}
$$

In the abstract form (1.2), when writing $\mathcal{W}=\mathscr{W} \circ E$ with $E$ denoting the linear operator $u \mapsto(e, w):=\left(e(u),\left.u\right|_{\Gamma}\right)$ and with $\left.u\right|_{\Gamma}$ denoting the trace of $u$ on the boundary $\Gamma$, this reads as

$$
\begin{array}{lll}
\mathcal{T}^{\prime} \dot{v}+E^{*} \Sigma=F(t) & \text { for } t \in(0, T], & \left.v\right|_{t=0}=v_{0}, \quad \text { and } \\
\dot{\Sigma}=\mathscr{W}^{\prime} E v+\dot{G}(t) & \text { for } t \in(0, T], & \left.\Sigma\right|_{t=0}=\Sigma_{0}:=\mathscr{W}^{\prime} E u_{0},
\end{array}
$$

where $E^{*}$ is the adjoint operator to $E$. The stored energy governing (1.5) is

$$
\mathscr{W}(e, w)=\int_{\Omega} \frac{1}{2} \mathbb{C} e: e \mathrm{~d} x+\int_{\Gamma} \frac{1}{2} \mathbb{B} w \cdot w \mathrm{~d} S
$$

while the external loading is now split into two parts acting differently, namely

$$
\langle F(t), u\rangle=\int_{\Omega} f(t) \cdot u \mathrm{~d} x \quad \text { and } \quad\langle G(t), w\rangle=\int_{\Gamma} g(t) \cdot w \mathrm{~d} x .
$$

Let us note that (1.6) involves the equation on $\Omega$, as well as, the equation on $\Gamma$. Thus $\mathcal{T}$ is to be understood as the functional on $\Omega \times \Gamma$, that is trivial on $\Gamma$ since no inertia is considered on the $(d-1)$-dimensional boundary $\Gamma$. In particular, the "generalized" stress $\Sigma=\mathscr{W}^{\prime} E u=\left(\mathbb{C} e(u),\left.\mathbb{B} u\right|_{\Gamma}\right)$ contains, besides the bulk stress tensor, also the traction stress vector. Relying on the linearity of $\mathscr{W}^{\prime}$, we have $\dot{\Sigma}=\mathscr{W}^{\prime} E v$ with $v=\dot{u}$, as used in (1.6b). Let us note that the adjoint operator $E^{*}:(\sigma, \varsigma) \mapsto \mathfrak{F}$ in $(1.6 \mathrm{a})$ with the traction force $\varsigma=\left.\mathbb{B} u\right|_{\Gamma}$ determines a bulk force $\mathfrak{F}$ as a functional on test displacements $u$ by

$$
\begin{aligned}
\int_{\Omega} \mathfrak{F} \cdot u \mathrm{~d} x=\langle\mathfrak{F}, u\rangle & =\left\langle E^{*}(\sigma, \varsigma), u\right\rangle=\langle(\sigma, \varsigma), E u\rangle=\left\langle(\sigma, \varsigma),\left(e(u),\left.u\right|_{\Gamma}\right)\right\rangle \\
& =\int_{\Omega} \sigma: e(u) \mathrm{d} x+\int_{\Gamma} \varsigma \cdot u \mathrm{~d} S=\int_{\Gamma}(\sigma \cdot \boldsymbol{n}+\varsigma) \cdot u \mathrm{~d} S-\int_{\Omega} \operatorname{div} \sigma \cdot u \mathrm{~d} x
\end{aligned}
$$

which clarifies the force $E^{*} \Sigma=\mathfrak{F}$ in (1.6a). The leap-frog time discretization of (1.6) then reads as

$$
\frac{\Sigma_{\tau h}^{k+1 / 2}-\Sigma_{\tau h}^{k-1 / 2}}{\tau}=\mathscr{W}_{h}^{\prime} E_{h} v_{\tau h}^{k}+D_{\tau h}^{k} \quad \text { and } \quad \mathcal{T}^{\prime} \frac{v_{\tau h}^{k+1}-v_{\tau h}^{k}}{\tau}+E_{h}^{*} \Sigma_{\tau h}^{k+1 / 2}=F_{\tau h}^{k+1 / 2}
$$

where $\mathscr{W}_{h}$ and $E_{h}$ are suitable FEM discretizations of $\mathscr{W}$ and $E$, cf. (3.1a) and (3.1c) below, and

$$
F_{\tau h}^{k+1 / 2}:=\frac{1}{\tau} \int_{k \tau}^{(k+1) \tau} F_{h}(t) \mathrm{d} t \quad \text { and } \quad D_{\tau h}^{k}:=\frac{1}{\tau} \int_{(k-1 / 2) \tau}^{(k+1 / 2) \tau} \dot{G}_{h}(t) \mathrm{d} t=\frac{G_{h}\left(\left(k+\frac{1}{2}\right) \tau\right)-G_{h}\left(\left(k-\frac{1}{2}\right) \tau\right)}{\tau} .
$$


As mentioned before, we assume here that $v$ is discretized in an element-wise constant way so that $\mathcal{T}$ leads to a diagonal matrix. In this case we do not need to employ numerical integration to approximate the mass matrix. For higher-order discretizations, however, mass lumping is necessary so as to obtain explicit discretization schemes. We refer to $[8,31,50]$ for details in the case $G \equiv 0$. The proposed FEM leads to a block diagonal matrix for $\mathcal{W}_{h}^{\prime}=E_{h}^{*} \mathscr{W}_{h}^{\prime} E_{h}$, which means that the resulting scheme does not require the solution of a big linear system at each iteration in time. The spatial FEM discretization exploits regularity available in linear elastodynamics, in particular that $\operatorname{div} \sigma$ and $e(v)$ in (1.5a) live in $L^{2}$-spaces. Moreover, the equations in (1.7) are decoupled in the sense that, first, $\Sigma_{\tau h}^{k+1 / 2}$ is calculated from the former equation and, second, $v_{\tau h}^{k+1}$ is calculated from the latter equation assuming, that $\left(v_{\tau h}^{k}, \Sigma_{\tau h}^{k-1 / 2}\right)$ is known from the previous time step. For $k=0$, it starts from $v_{\tau h}^{0}=v_{0}$ and from a half time step $\Sigma_{\tau h}^{1 / 2}=\Sigma_{\tau h}^{0}+\frac{\tau}{2} \mathscr{W}_{h}^{\prime} E_{h} v_{\tau h}^{0}$. For the space discretization, the lower order $Q_{k+1}^{\text {div }}-Q_{k}$ finite element is obtained for $k=0$ and in this case the velocity is discretized as piecewise constant on rectangular or cubic elements while the stress is discretized by piecewise bi-linear functions with some continuities. Namely the normal component of the stress is continuous across edges of adjacent elements while the tangential component is allowed to be discontinuous. For more details about the space discretization we refer the interested reader to [8]. Alternative discretizations for the linear elasticity problem have been proposed by D. Arnold and his collaborators who designed mixed finite elements for general rectangular and triangular grids [4-6]. For tetrahedral leap-frog discretization of the elastodynamics see [21]. In general, the leap-frog scheme has been frequently used in geophysics to calculate seismic wave propagation with the finite differences method, cf. e.g. $[13,25,51]$.

When taking the average (i.e. the sum with the weights $\frac{1}{2}$ and $\frac{1}{2}$ ) of the second equation in (1.7) in the level $k$ and $k-1$ tested by $v_{\tau h}^{k}$ and summing it with the first equation in (1.7) tested by $\left[\mathscr{W}_{h}^{\prime \prime}\right]^{-1}\left(\Sigma_{\tau h}^{k+1 / 2}+\Sigma_{\tau h}^{k-1 / 2}\right) / 2$, we obtain

$$
\begin{gathered}
\frac{1}{2 \tau}\left\langle\left[\mathscr{W}_{h}^{\prime}\right]^{-1} \Sigma_{\tau h}^{k+1 / 2}, \Sigma_{\tau h}^{k+1 / 2}\right\rangle-\frac{1}{2 \tau}\left\langle\left[\mathscr{W}_{h}^{\prime}\right]^{-1} \Sigma_{\tau h}^{k-1 / 2}, \Sigma_{\tau h}^{k-1 / 2}\right\rangle \\
=\left\langle\frac{\Sigma_{\tau h}^{k+1 / 2}+\Sigma_{\tau h}^{k-1 / 2}}{2}, E_{h} v_{\tau h}^{k}\right\rangle+\left\langle\left[\mathscr{W}_{h}^{\prime}\right]^{-1} D_{\tau h}^{k}, \frac{\sum_{\tau h}^{k+1 / 2}+\Sigma_{\tau h}^{k-1 / 2}}{2}\right\rangle \text { and } \\
\left\langle\mathcal{T}^{\prime} \frac{v_{\tau h}^{k+1}-v_{\tau h}^{k-1}}{\tau}, v_{\tau h}^{k}\right\rangle+\left\langle\frac{\Sigma_{\tau h}^{k+1 / 2}+\Sigma_{\tau h}^{k-1 / 2}}{2}, E_{h} v_{\tau h}^{k}\right\rangle=\left\langle\frac{F_{\tau h}^{k+1 / 2}+F_{\tau h}^{k-1 / 2}}{2}, v_{\tau h}^{k}\right\rangle .
\end{gathered}
$$

Summing it up, we get that the following discrete energy is conserved

$$
\frac{1}{2}\left\langle\mathcal{T}^{\prime} v_{\tau h}^{k+1}, v_{\tau h}^{k}\right\rangle+\Phi_{h}\left(\Sigma_{\tau h}^{k+1 / 2}\right) \quad \text { with } \quad \Phi_{h}(\Sigma)=\frac{1}{2}\left\langle\left[\mathscr{W}_{h}^{\prime}\right]^{-1} \Sigma, \Sigma\right\rangle
$$

Note that $\Phi_{h}$ is the discrete stored energy expressed in terms of the generalized stress. In contrast to (1.3), this formulation allows for enhancement of the discrete stored energy by some internal variables. The energy (1.9) is shown to be a positive quantity under the following CFL condition

$$
\left\langle\left[\mathcal{W}_{h}^{\prime}\right]^{-1} \Sigma_{h}, \Sigma_{h}\right\rangle \geq \frac{\tau^{2}}{4}\left\langle E_{h}^{*} \Sigma_{h},\left(\mathcal{T}^{\prime}\right)^{-1} E_{h}^{*} \Sigma_{h}\right\rangle
$$

for any $\Sigma_{h}$ from the respective finite-dimensional subspace. Moreover, $F=0$ is often considered, which makes the a priori estimation easier. Let us also note that the adjective "leap-frog" is sometimes used also for the time-discretization (1.3) if written as a two-step scheme, cf. e.g. ([19], Sect. 7.1.1.1).

The plan of this article is as follows: In Section 2, we complement the abstract system (1.6) by another equation for some internal variable and cast its weak formulation without relying on any regularity. Then, in Section 3, we extend the two-step leap-frog discrete scheme (1.7) to a suitable three-step scheme, and study the energy properties of the proposed scheme. Then, in Section 4, we prove the numerical stability of the 3-step staggered approximation scheme and its convergence under the CFL condition (4.1). Such an abstract scheme is then illustrated in Section 5 on several examples from continuum mechanics, in particular on models of 
plasticity, creep, diffusion, and damage. For illustration of computational efficiency, we refer to [44] where this scheme was implemented for another problem, namely a delamination (i.e. interfacial damage).

It should be emphasized that, to the best of our knowledge, a rigorously justified (as far as numerical stability and convergence) combination of the explicit staggered discretization with nonlinear dissipative processes on some internal variables is new, although occasionally some dissipative nonlinear phenomena can be found in literature as in [45] for a unilateral contact, in [13] for a Maxwell viscoelastic rheology, in [47] for electroactive polymers, in [23] for an aeroelastic system, or in [24] for general thermomechanical systems, but without any numerical stability (a priori estimates) and convergence guaranteed.

\section{INTERNAL VARIABLES AND THEIR DISSIPATIVE EVOLUTION.}

The concept of internal variables has a long tradition, $c f$. [36], and opens wide options for material modelling, cf. e.g. $[35,37]$ and references therein. Typically, internal variables are governed by a parabolic-type 1st-order evolution. The abstract system (1.2) is thus generalized to

$$
\begin{array}{ll}
\mathcal{T}^{\prime} \ddot{u}+\mathcal{W}_{u}^{\prime}(u, z)=F(t) & \text { for } t \in(0, T],\left.\quad u\right|_{t=0}=u_{0},\left.\quad \dot{u}\right|_{t=0}=v_{0}, \\
\partial \Psi(\dot{z})+\mathcal{W}_{z}^{\prime}(u, z) \ni 0 & \text { for } t \in(0, T],\left.\quad z\right|_{t=0}=z_{0} .
\end{array}
$$

The inclusion in (2.1b) refers to a possibility that the convex (pseudo)potential $\Psi$ of dissipative forces may be nonsmooth and then its subdifferential $\partial \Psi$ can be multivalued.

Combination of the 2nd-order evolution (1.2) with such 1st-order evolution is to be handled carefully. In contrast to the implicit staggered schemes, $c f$. [41], the constitutive equation is differentiated in time, $c f$. (1.5a), and it seems necessary to use the staggered scheme so that the internal-variable flow rule can be used without being differentiated in time, even for a quadratic stored energy $\mathcal{W}$.

Moreover, to imitate the leap-frog scheme, it seems suitable (or maybe even necessary) that the stored energy $\mathcal{W}$ may be expressed in terms of the generalized stress as

$$
\mathcal{W}(u, z)=\Phi(\Sigma, z) \quad \text { with } \quad \Sigma=\mathfrak{C} E u, \text { and } \Phi(\cdot, z) \text { and } \Phi(\Sigma, \cdot) \text { quadratic, }
$$

where $\mathfrak{C}$ stands for a "generalized" elasticity tensor and $E$ is an abstract gradient-type operator. Typically $E u=\left(e(u),\left.u\right|_{\Gamma}\right)$ or simply $E u=e(u)$ are considered here in the context of continuum mechanics at small strains, $c f$. the examples in Section 5. Here, $\Sigma$ may not directly enter the balance of forces and is thus to be called rather as some "proto-stress", while the actual generalized stress will be denoted by $S$. For a relaxation of the last requirement of (2.2) see Remark 4.4 below. Then, likewise (1.6), we can write the system (2.1) in the velocity/proto-stress formulation as

$$
\begin{aligned}
& \dot{\Sigma}=\mathfrak{C} E v+\dot{G}(t) \\
& \mathcal{T}^{\prime} \dot{v}+E^{*} S=F(t) \quad \text { with } \quad S=\mathfrak{C}^{*} \Phi_{\Sigma}^{\prime}(\Sigma, z) \\
& \partial \Psi(\dot{z})+\Phi_{z}^{\prime}(\Sigma, z) \ni 0 \\
& \left.\Sigma\right|_{t=0}=\Sigma_{0}:=\mathfrak{C} E u_{0}+G(0),\left.\quad v\right|_{t=0}=v_{0},\left.\quad z\right|_{t=0}=z_{0} .
\end{aligned}
$$

Here $\Phi_{\Sigma}^{\prime}(\Sigma, z)$ is a "generalized" strain and, when multiplied by $\mathfrak{C}^{*}$, it becomes a generalized stress. Actually, (1.6) is a special case of (2.3) when $\Phi=\Phi(\Sigma)=\int_{\Omega} \frac{1}{2} \mathbb{C}^{-1} \sigma: \sigma \mathrm{d} x+\int_{\Gamma} \mathbb{B}^{-1} \varsigma \cdot \varsigma \mathrm{d} S$ and $S=\Sigma=(\sigma, \varsigma)$ while $\mathfrak{C}=\mathscr{W}^{\prime}=(\mathbb{C}, \mathbb{B})$, so that indeed $S=\mathfrak{C}^{*} \Phi_{\Sigma}^{\prime}(\Sigma)=(\mathbb{C}, \mathbb{B})\left(\mathbb{C}^{-1} \sigma, \mathbb{B}^{-1} \varsigma\right)=(\sigma, \varsigma)=\Sigma$.

The energy properties of this system can be revealed by testing the particular equations/inclusions in (2.3) by $\Phi_{\Sigma}^{\prime}(\Sigma, z), v$, and $\dot{z}$. Thus, at least formally, we obtain

$$
\begin{aligned}
& \left\langle\Phi_{\Sigma}^{\prime}(\Sigma, z), \dot{\Sigma}\right\rangle=\left\langle\Phi_{\Sigma}^{\prime}(\Sigma, z), \mathfrak{C} E v+\dot{G}\right\rangle=\left\langle\mathfrak{C}^{*} \Phi_{\Sigma}^{\prime}(\Sigma, z), E v\right\rangle+\left\langle\Phi_{\Sigma}^{\prime}(\Sigma, z), \dot{G}\right\rangle, \\
& \left\langle\mathcal{T}^{\prime} \dot{v}, v\right\rangle+\left\langle\mathfrak{C}^{*} \Phi_{\Sigma}^{\prime}(\Sigma, z), E v\right\rangle=\langle F(t), v\rangle, \\
& \Xi(\dot{z})+\left\langle\Phi_{z}^{\prime}(\Sigma, z), \dot{z}\right\rangle \leq 0 \quad \text { with } \quad \Xi(\dot{z}):=\inf \langle\partial \Psi(\dot{z}), \dot{z}\rangle .
\end{aligned}
$$


The functional $\Xi$ is a dissipative rate and the "inf" in it refers to the fact that the dissipative potential $\Psi$ can be nonsmooth and thus the subdifferential $\partial \Psi$ can be multivalued even at $\dot{z} \neq 0$, otherwise an equality in (2.4c) holds. Summing it up and using the calculus $\frac{\mathrm{d}}{\mathrm{d} t} \mathcal{T}(v)=\left\langle\mathcal{T}^{\prime} v, \dot{v}\right\rangle=\left\langle\mathcal{T}^{\prime} \dot{v}, v\right\rangle$ and $\frac{\mathrm{d}}{\mathrm{d} t} \Phi(\Sigma, z)=\left\langle\Phi_{\Sigma}^{\prime}(\Sigma, z), \dot{\Sigma}\right\rangle+$ $\left\langle\Phi_{z}^{\prime}(\Sigma, z), \dot{z}\right\rangle$, we obtain the following inequality for the energy,

$$
\frac{\mathrm{d}}{\mathrm{d} t} \underbrace{(I(v)+\Phi(\Sigma, z)}_{\begin{array}{c}
\text { kinetic and } \\
\text { stored energies }
\end{array}}+\underbrace{\Xi(\dot{z})}_{\begin{array}{c}
\text { dissipation } \\
\text { rate }
\end{array}} \leq \underbrace{\langle F(t), v\rangle+\left\langle\Phi_{\Sigma}^{\prime}(\Sigma, z), \dot{G}\right\rangle}_{\begin{array}{c}
\text { power of } \\
\text { external force }
\end{array}} .
$$

Actually, (2.4c) and (2.5) often hold as equalities.

Let us now formulate some abstract functional setting of the system (2.3). For some Banach spaces $\mathcal{S}, \mathcal{Z}$, and $\mathcal{Z}_{1} \supset \mathcal{Z}$ and for a Hilbert space $\mathcal{H}$, let $\Phi: \mathcal{S} \times \mathcal{Z} \rightarrow \mathbb{R}$ be smooth and coercive, $\mathcal{T}: \mathcal{H} \rightarrow \mathbb{R}$ be quadratic and coercive, and let $\Psi: \mathcal{Z} \rightarrow[0,+\infty]$ be convex, lower semicontinuous, and coercive on $\mathcal{Z}_{1}, c f$. (4.2) below. Intentionally, we do not want to rely on any regularity which is usually at disposal in linear problems but might be restrictive in some nonlinear problems. For this reason, we reconstruct the abstract "displacement" and use (2.3a) integrated in time, i.e.

$$
\Sigma=\mathfrak{C} E u+G \quad \text { with } \quad u(t):=\int_{0}^{t} v(t) \mathrm{d} t+u_{0}
$$

Moreover, we still need another Banach space $\mathcal{E}$ and define the Banach space $\mathcal{U}:=\{u \in \mathcal{H} ; E u \in \mathcal{E}\}$ equipped with the standard graph norm. Then, by definition, we have the continuous embedding $\mathcal{U} \rightarrow \mathcal{H}$ and the continuous linear operator $E: \mathcal{U} \rightarrow \mathcal{E}$. We assume that $\mathcal{U}$ is embedded into $\mathcal{H}$ densely, so that $\mathcal{H}^{*} \subset \mathcal{U}^{*}$ and that $\mathcal{H}$ is identified with its dual $\mathcal{H}^{*}$, so that we have the so-called Gelfand triple

$$
\mathcal{U} \subset \mathcal{H} \cong \mathcal{H}^{*} \subset \mathcal{U}^{*}
$$

We further consider the abstract elasticity tensor $\mathfrak{C}$ as a linear continuous operator $\mathcal{E} \rightarrow \mathcal{S}$. Therefore $\mathfrak{C} E u \in \mathcal{S}$ provided $u \in \mathcal{U}$ so that the equation (2.6) is meant in $\mathcal{S}$ and one needs $G(t) \in \mathcal{S}$. Let us note that $\mathcal{T}^{\prime}: \mathcal{H} \rightarrow$ $\mathcal{H}^{*} \cong \mathcal{H}, \Phi_{\Sigma}^{\prime}: \mathcal{S} \times \mathcal{Z} \rightarrow \mathcal{S}^{*}, E^{*}: \mathcal{E}^{*} \rightarrow \mathcal{U}^{*}$, and $\mathfrak{C}^{*}: \mathcal{S}^{*} \rightarrow \mathcal{E}^{*}$, so that $\mathcal{T}^{\prime} v \in \mathcal{H}^{*}$ provided $v \in \mathcal{H}$ and also $S=\mathfrak{C}^{*} \Phi_{\Sigma}^{\prime} \in \mathcal{E}^{*}$ and $E^{*} S \in \mathcal{H}^{*}$. In particular, the equation (2.3b) can be meant in $\mathcal{H}$ if integrated in time, and one needs $F(t)$ valued in $\mathcal{H}$.

For a Banach space $\mathcal{X}$, we will use the standard notation $L^{p}(0, T ; \mathcal{X})$ for Bochner spaces of the Bochner measurable functions $[0, T] \rightarrow \mathcal{X}$ whose norm is integrable with the power $p$ or essentially bounded if $p=$ $\infty$, and $W^{1, p}(0, T ; \mathcal{X})$ the space of functions from $L^{p}(0, T ; \mathcal{X})$ whose distributional time derivative is also in $L^{p}(0, T ; \mathcal{X})$. Also, $C^{k}(0, T ; \mathcal{X})$ will denote the space of functions $[0, T] \rightarrow \mathcal{X}$ whose $k$ th-derivative is continuous, and $C_{\mathrm{w}}(0, T ; \mathcal{X})$ will denote the space of weakly continuous functions $[0, T] \rightarrow \mathcal{X}$. Later, we will also use $\operatorname{Lin}(\mathcal{U}, \mathcal{E})$, denoting the space of linear bounded operators $\mathcal{U} \rightarrow \mathcal{E}$ normed by the usual sup-norm.

A weak formulation of (2.3b) can be obtained after by-part integration over the time interval $I=[0, T]$ when tested by a smooth function. It is often useful to consider

$$
\Phi(\Sigma, z)=\Phi_{0}(\Sigma, z)+\Phi_{1}(z) \quad \text { with }\left[\Phi_{0}\right]_{z}^{\prime}: \mathcal{S} \times \mathcal{Z} \rightarrow \mathcal{Z}_{1}^{*} \text { and } \Phi_{1}^{\prime}: \mathcal{Z} \rightarrow \mathcal{Z}^{*}
$$

and to use integration by-parts for the term $\left\langle\Phi_{1}^{\prime}(z), \dot{z}\right\rangle$. We thus arrive to the following definition.

Definition 2.1 (Weak solution to (2.3)). The quadruple $(u, \Sigma, v, z) \in C_{\mathrm{w}}(0, T ; \mathcal{U}) \times C_{\mathrm{w}}(0, T ; \mathcal{S}) \times C_{\mathrm{w}}(0, T ; \mathcal{H}) \times$ $C_{\mathrm{w}}(0, T ; \mathcal{Z})$ with $\Psi(\dot{z}) \in L^{1}(I)$ and $\dot{z} \in L^{1}\left(0, T ; \mathcal{Z}_{1}\right)$ will be called a weak solution to the initial-value problem (2.3) with (2.6) if $v=\dot{u}$ in the distributional sense, $\Sigma=\mathfrak{C} E u+G$ holds a.e. on $I$, and if

$$
\int_{0}^{T}\left\langle\Phi_{\Sigma}^{\prime}(\Sigma, z), \mathfrak{C} E \widetilde{v}\right\rangle_{\mathcal{S}^{*} \times \mathcal{S}}-\left\langle\mathcal{T}^{\prime} v, \dot{\widetilde{v}}\right\rangle_{\mathcal{H}^{*} \times \mathcal{H}} \mathrm{d} t=\left\langle\mathcal{T}^{\prime} v_{0}, \widetilde{v}(0)\right\rangle_{\mathcal{H}^{*} \times \mathcal{H}}+\int_{0}^{T}\langle F, \widetilde{v}\rangle_{\mathcal{H}^{*} \times \mathcal{H}} \mathrm{d} t
$$


for any $\widetilde{v} \in C^{1}(0, T ; \mathcal{H}) \cap C(0, T ; \mathcal{U})$ with $\widetilde{v}(T)=0$, and

$$
\int_{0}^{T} \Psi(\widetilde{z})+\left\langle\left[\Phi_{0}\right]_{z}^{\prime}(\Sigma, z), \widetilde{z}-\dot{z}\right\rangle_{\mathcal{Z}_{1}^{*} \times \mathcal{Z}_{1}}+\left\langle\Phi_{1}^{\prime}(z), \widetilde{z}\right\rangle_{\mathcal{Z}^{*} \times \mathcal{Z}} \mathrm{d} t+\Phi_{1}\left(z_{0}\right) \geq \Phi_{1}(z(T))+\int_{0}^{T} \Psi(\dot{z}) \mathrm{d} t
$$

for any $\widetilde{z} \in C(0, T ; \mathcal{Z})$, where indices in the dualities $\langle\cdot, \cdot\rangle$ indicate the respective spaces in dualities, and if also $u(0)=u_{0}, \Sigma(0)=\Sigma_{0}$, and $z(0)=z_{0}$.

Let us note that the remaining initial condition $v(0)=v_{0}$ is contained in (2.8a). Definition 2.1 works successfully for $p>1$, i.e. for rate-dependent evolution of the abstract internal variable $z$, so that $\dot{z} \in L^{p}\left(0, T ; \mathcal{Z}_{1}\right)$. For the rate-dependent evolution when $p=1$, we would need to modify it. Here, we restrict ourselves to $p \geq 2$, because of the a priori estimates in Proposition 4.1.

\section{A threE-STEP STAGGERED TIME DisCRETIZATION}

We derive in this section the leap-frog discretization of (2.3a) and (2.3b) combined with an implicit discretization for (2.3c), using a fractional-step split (called also a staggered scheme) with a mid-point formula for (2.3c). Instead of a two-step scheme (1.7), we obtain a three-step scheme and therefore, from now on, we abandon the convention of a half-step notation as used in (1.7) and write $k+1$ instead of $k+1 / 2$.

To this aim, we consider sequences of nested finite-dimensional subspaces $S_{h} \subset \mathcal{S}, V_{h} \subset \mathcal{H}$, and $Z_{h} \subset \mathcal{Z}$ where the values of the respective discrete variables $\Sigma_{h}, v_{h}$, and $z_{h}$ will be, assuming that their unions are dense in the respective Banach spaces. We will use an interpolation operator $I_{h}: \operatorname{Lin}\left(\mathcal{S}, S_{h}\right)$ and the embedding operator $J_{h}: Z_{h} \rightarrow \mathcal{Z}$; it is important that the collection $\left\{J_{h}\right\}_{h>0}$ is uniformly bounded and, since $\bigcup_{h>0} Z_{h}$ is dense in $\mathcal{Z}$, the sequence $\left\{J_{h}\right\}_{h>0}$ converges to the identity on $\mathcal{Z}$ strongly. We consider $E_{h} \in \operatorname{Lin}\left(V_{h}, \mathcal{E}\right)$. Let us note that we allow for a "non-conformal" approximation of $v$, i.e. $V_{h} \subset \mathcal{H}$ is not necessarily a subspace of $\mathcal{U}$. This is in agreement with discretizations of the velocity as in $[7,8,11,18,50]$.

Considering that we know from previous step $\Sigma_{\tau h}^{k}, v_{\tau h}^{k}, z_{\tau h}^{k}$, then the proposed discretization scheme is

$$
\begin{aligned}
\text { (1) calculate } \Sigma_{\tau h}^{k+1}: & \frac{\Sigma_{\tau h}^{k+1}-\Sigma_{\tau h}^{k}}{\tau}=I_{h} \mathfrak{C} E_{h} v_{\tau h}^{k}+D_{\tau h}^{k}, \\
\text { (2) calculate } z_{\tau h}^{k+1}: & J_{h}^{*} \partial \Psi\left(\frac{z_{\tau h}^{k+1}-z_{\tau h}^{k}}{\tau}\right)+\Phi_{z}^{\prime}\left(\Sigma_{\tau h}^{k+1}, \frac{z_{\tau h}^{k+1}+z_{\tau h}^{k}}{2}\right) \ni 0, \\
\text { (3) calculate } v_{\tau h}^{k+1}: & \mathcal{T}^{\prime} \frac{v_{\tau h}^{k+1}-v_{\tau h}^{k}}{\tau}+E_{h}^{*} S_{\tau h}^{k+1}=F_{\tau h}^{k+1 / 2} \text { with } S_{\tau h}^{k+1}=\mathfrak{C}^{*} I_{h}^{*} \Phi_{\Sigma}^{\prime}\left(\Sigma_{\tau h}^{k+1}, z_{\tau h}^{k+1}\right), \\
\text { and } u_{\tau h}^{k+1}: & u_{\tau h}^{k+1}=u_{\tau h}^{k}+\tau v_{\tau h}^{k+1},
\end{aligned}
$$

where $F_{\tau h}^{k+1 / 2}$ and $D_{\tau h}^{k}$ are from (1.8). It seems important in the non-linear case to compute the variables in the order given above. We note however, that for the linear viscoelastic problem with Maxwell rheology a scheme with a different ordering has been proposed in Part I, Section 2 of [27]. The potentials $\Phi, \Psi$, and $\mathcal{T}$ are considered restricted on $S_{h} \times Z_{h}, Z_{h}$, and $V_{h}$, so that their corresponding (sub)differentials $\Phi_{\Sigma}^{\prime}, \Phi_{z}^{\prime}, \partial \Psi$, and $\mathcal{T}^{\prime}$ are valued in $S_{h}^{*}, Z_{h}^{*}$, and $V_{h}^{*}$, respectively. The particular equations/inclusion in (3.1) are thus to be understood in $S_{h}, Z_{h}^{*}, V_{h}^{*}, \mathcal{E}^{*}$, and $V_{h}$, respectively. The only implicit equation is (3.1b). Note however that even this equation becomes explicit if there are no spatial gradients in $\Phi$ and $\Psi$. In view of the definition of the convex subdifferential, (3.1b) means the variational inequality

$$
\Psi(\widetilde{z})+\left\langle\Phi_{z}^{\prime}\left(\Sigma_{\tau h}^{k+1}, \frac{z_{\tau h}^{k+1}+z_{\tau h}^{k}}{2}\right), \widetilde{z}-\frac{z_{\tau h}^{k+1}-z_{\tau h}^{k}}{\tau}\right\rangle \geq \Psi\left(\frac{z_{\tau h}^{k+1}-z_{\tau h}^{k}}{\tau}\right)
$$


for any $\widetilde{z} \in V_{h}$. In any case, the equation (3.1b) posseses a potential

$$
z \mapsto \frac{2}{\tau} \Phi\left(\Sigma_{\tau h}^{k+1}, \frac{z+z_{\tau h}^{k}}{2}\right)+\Psi\left(\frac{z-z_{\tau h}^{k}}{\tau}\right)
$$

which is to be minimized on $Z_{h}$. Therefore the existence of a solution to this inclusion (3.1b), or equivalently of the variational inequality (3.2), can be shown by a direct method, $c f$. also [41]. The scheme (3.1) is thus to be solved recurrently for $k=0,1, \ldots, T / \tau-1$, starting from the initial conditions (2.3d) assumed, for simplicity, to live in the respective finite-dimensional spaces.

The energy properties of this scheme can be obtained by imitating (2.4) and (2.5). More specifically, we proceed as follows: we test (3.1a) by $\frac{1}{2} \Phi_{\Sigma}^{\prime}\left(\sum_{\tau h}^{k+1}, z_{\tau h}^{k+1}\right)+\frac{1}{2} \Phi_{\Sigma}^{\prime}\left(\sum_{\tau h}^{k}, z_{\tau h}^{k}\right)$, then test (3.1b) by $\frac{z_{\tau h}^{k+1}-z_{\tau h}^{k}}{\tau}$, and eventually test the average of (3.1c) at the level $k+1$ and $k$ by $v_{\tau h}^{k}$. Using that $\Phi(\cdot, z)$ and $\Phi(\Sigma, \cdot)$ are quadratic as assumed in $(2.2)$, we have

$$
\begin{aligned}
\langle & \left.\frac{\Phi_{\Sigma}^{\prime}\left(\sum_{\tau h}^{k+1}, z_{\tau h}^{k+1}\right)+\Phi_{\Sigma}^{\prime}\left(\sum_{\tau h}^{k}, z_{\tau h}^{k}\right)}{2}, \frac{\sum_{\tau h}^{k+1}-\Sigma_{\tau h}^{k}}{\tau}\right\rangle \\
= & \left\langle\frac{\Phi_{\Sigma}^{\prime}\left(\sum_{\tau h}^{k+1}, z_{\tau h}^{k}\right)+\Phi_{\Sigma}^{\prime}\left(\sum_{\tau h}^{k}, z_{\tau h}^{k}\right)}{2}, \frac{\sum_{\tau h}^{k+1}-\Sigma_{\tau h}^{k}}{\tau}\right\rangle \\
& +\frac{\tau}{2}\left\langle\frac{\Phi_{\Sigma}^{\prime}\left(\Sigma_{\tau h}^{k+1}, z_{\tau h}^{k+1}\right)-\Phi_{\Sigma}^{\prime}\left(\sum_{\tau h}^{k+1}, z_{\tau h}^{k}\right)}{\tau}, \frac{\sum_{\tau h}^{k+1}-\Sigma_{\tau h}^{k}}{\tau}\right\rangle \\
= & \frac{\Phi\left(\sum_{\tau h}^{k+1}, z_{\tau h}^{k}\right)-\Phi\left(\Sigma_{\tau h}^{k}, z_{\tau h}^{k}\right)}{\tau}+\frac{\tau}{2}\left\langle\frac{\Phi_{\Sigma}^{\prime}\left(\sum_{\tau h}^{k+1}, z_{\tau h}^{k+1}\right)-\Phi_{\Sigma}^{\prime}\left(\Sigma_{\tau h}^{k+1}, z_{\tau h}^{k}\right)}{\tau}, \frac{\sum_{\tau h}^{k+1}-\Sigma_{\tau h}^{k}}{\tau}\right\rangle,
\end{aligned}
$$

where we used also (3.1a), and

$$
\left\langle\Phi_{z}^{\prime}\left(\Sigma_{\tau h}^{k+1}, \frac{z_{\tau h}^{k+1}+z_{\tau h}^{k}}{2}\right), \frac{z_{\tau h}^{k+1}-z_{\tau h}^{k}}{\tau}\right\rangle=\frac{\Phi\left(\sum_{\tau h}^{k+1}, z_{\tau h}^{k+1}\right)-\Phi\left(\Sigma_{\tau h}^{k+1}, z_{\tau h}^{k}\right)}{\tau} .
$$

Therefore, using again the particular equations/inclusion in (3.1), we get respectively

$$
\begin{aligned}
& \frac{\Phi\left(\Sigma_{\tau h}^{k+1}, z_{\tau h}^{k}\right)-\Phi\left(\Sigma_{\tau h}^{k}, z_{\tau h}^{k}\right)}{\tau}=\left\langle\frac{\Phi_{\Sigma}^{\prime}\left(\Sigma_{\tau h}^{k+1}, z_{\tau h}^{k+1}\right)+\Phi_{\Sigma}^{\prime}\left(\Sigma_{\tau h}^{k}, z_{\tau h}^{k}\right)}{2}, I_{h} \mathfrak{C} E_{h} v_{\tau h}^{k}+D_{\tau h}^{k}\right\rangle \\
& -\frac{\tau}{2}\left\langle\frac{\Phi_{\Sigma}^{\prime}\left(\Sigma_{\tau h}^{k+1}, z_{\tau h}^{k+1}\right)-\Phi_{\Sigma}^{\prime}\left(\sum_{\tau h}^{k+1}, z_{\tau h}^{k}\right)}{\tau}, \frac{\sum_{\tau h}^{k+1}-\Sigma_{\tau h}^{k}}{\tau}\right\rangle, \\
& \Xi\left(\frac{z_{\tau h}^{k+1}-z_{\tau h}^{k}}{\tau}\right)+\frac{\Phi\left(\sum_{\tau h}^{k+1}, z_{\tau h}^{k+1}\right)-\Phi\left(\sum_{\tau h}^{k+1}, z_{\tau h}^{k}\right)}{\tau} \leq 0, \quad \text { and } \\
& \left\langle\mathcal{T}^{\prime} \frac{v_{\tau h}^{k+1}-v_{\tau h}^{k-1}}{2 \tau}, v_{\tau h}^{k}\right\rangle+\left\langle E_{h}^{*} \mathfrak{C}^{*} I_{h}^{*} \frac{\Phi_{\Sigma}^{\prime}\left(\Sigma_{\tau h}^{k+1}, z_{\tau h}^{k+1}\right)+\Phi_{\Sigma}^{\prime}\left(\Sigma_{\tau h}^{k}, z_{\tau h}^{k}\right)}{2}, v_{\tau h}^{k}\right\rangle=\left\langle F_{\tau h}^{k+1 / 2}, v_{\tau h}^{k}\right\rangle .
\end{aligned}
$$

Let us also note that, if $\Psi(0)=0$ is assumed, the substitution $\widetilde{z}=0$ into the inequality (3.2) gives $\Psi\left(\frac{z_{\tau h}^{k+1}-z_{\tau h}^{k}}{\tau}\right)$ instead of the dissipation rate $\Xi\left(\frac{z_{\tau h}^{k+1}-z_{\tau h}^{k}}{\tau}\right)$ in (3.5b), which is a suboptimal estimate except if $\Psi$ is degree-1 positively homogeneous.

Summing (3.5) up, we benefit from the cancellation of the terms $\pm \Phi\left(\Sigma_{\tau h}^{k+1}, z_{\tau h}^{k}\right)$, which is the usual goal and attribute of well-designed fractional-split schemes. Thus, using also the simple algebra

$$
\left\langle\mathcal{T}^{\prime}\left(v_{\tau h}^{k+1}-v_{\tau h}^{k-1}\right), v_{\tau h}^{k}\right\rangle=\left\langle\mathcal{T}^{\prime} v_{\tau h}^{k+1}, v_{\tau h}^{k}\right\rangle-\left\langle\mathcal{T}^{\prime} v_{\tau h}^{k}, v_{\tau h}^{k-1}\right\rangle,
$$


we obtain the analog of (2.5), namely

$$
\begin{gathered}
\frac{\left\langle\mathcal{T}^{\prime} v_{\tau h}^{k+1}, v_{\tau h}^{k}\right\rangle-\left\langle\mathcal{T}^{\prime} v_{\tau h}^{k}, v_{\tau h}^{k-1}\right\rangle}{2 \tau}+\frac{\Phi\left(\sum_{\tau h}^{k+1}, z_{\tau h}^{k+1}\right)-\Phi\left(\Sigma_{\tau h}^{k}, z_{\tau h}^{k}\right)}{\tau}+\Xi\left(\frac{z_{\tau h}^{k+1}-z_{\tau h}^{k}}{\tau}\right) \\
\leq\left\langle F_{\tau h}^{k+1 / 2}, v_{\tau h}^{k}\right\rangle+\left\langle\frac{\Phi_{\Sigma}^{\prime}\left(\sum_{\tau h}^{k+1}, z_{\tau h}^{k+1}\right)+\Phi_{\Sigma}^{\prime}\left(\Sigma_{\tau h}^{k}, z_{\tau h}^{k}\right)}{2}, D_{\tau h}^{k}\right\rangle \\
-\frac{\tau}{2}\left\langle\frac{\Phi_{\Sigma}^{\prime}\left(\Sigma_{\tau h}^{k+1}, z_{\tau h}^{k+1}\right)-\Phi_{\Sigma}^{\prime}\left(\Sigma_{\tau h}^{k+1}, z_{\tau h}^{k}\right)}{\tau}, \frac{\sum_{\tau h}^{k+1}-\Sigma_{\tau h}^{k}}{\tau}\right\rangle .
\end{gathered}
$$

If $\Psi$ is smooth except possibly at zero, there is even equality in (3.7).

Considering some approximate values $\left\{z_{\tau h}^{k}\right\}_{k=0, \ldots, K}$ of the variable $z$ with $K=T / \tau$, we define the piecewiseconstant and the piecewise affine interpolants respectively by

$$
\begin{array}{llrl}
\bar{z}_{\tau h}(t) & =z_{\tau h}^{k}, \quad \bar{z}_{\tau h}(t)=\frac{1}{2} z_{\tau h}^{k}+\frac{1}{2} z_{\tau h}^{k-1}, & & \text { and } \\
z_{\tau h}(t)=\frac{t-(k-1) \tau}{\tau} z_{\tau h}^{k}+\frac{k \tau-t}{\tau} z_{\tau h}^{k-1} & & \text { for }(k-1) \tau<t \leq k \tau .
\end{array}
$$

Similar meaning is implied for $\Sigma_{\tau h}, v_{\tau h}, \bar{\Sigma}_{\tau h}, \bar{v}_{\tau h}, \bar{F}_{\tau h}$, etc. The discrete scheme (3.1) can be written in a "compact" form as

$$
\begin{aligned}
& \dot{\Sigma}_{\tau h}=I_{h} \mathfrak{C} E_{h} \bar{v}_{\tau h}+\dot{G}_{\tau h} \quad \text { and } \quad \dot{u}_{\tau h}=\bar{v}_{\tau h}, \\
& J_{h}^{*} \partial \Psi\left(\dot{z}_{\tau h}\right)+\Phi_{z}^{\prime}\left(\bar{\Sigma}_{\tau h}, \bar{z}_{\tau h}\right) \ni 0, \\
& \mathcal{T}^{\prime} \dot{v}_{\tau h}+E_{h}^{*} \bar{S}_{\tau h}=\bar{F}_{\tau h} \quad \text { with } \quad \bar{S}_{\tau h}=\mathfrak{C}^{*} I_{h}^{*} \Phi_{\Sigma}^{\prime}\left(\bar{\Sigma}_{\tau h}, \bar{z}_{\tau h}\right)
\end{aligned}
$$

to be valid a.e. on the time interval $[0, T]$.

\section{Numerical Stability AND CONVERGEnCE}

Because the energy (1.9) involves now also the internal variable, the CFL condition has to be modified. More specifically, we assume that

$$
\exists \eta>0 \forall \Sigma_{h} \in S_{h}, z_{h} \in Z_{h}: \quad \Phi\left(\Sigma_{h}, z_{h}\right) \geq \frac{\tau^{2}}{4-\eta}\left\langle E_{h}^{*} S_{h},\left(\mathcal{T}^{\prime}\right)^{-1} E_{h}^{*} S_{h}\right\rangle_{\mathcal{H}^{*} \times \mathcal{H}} \quad \text { with } S_{h}=\mathfrak{C}^{*} I_{h}^{*} \Phi_{\Sigma}^{\prime}\left(\Sigma_{h}, z_{h}\right),
$$

where $\Sigma_{h}$ and $z_{h}$ are considered from the corresponding finite-dimensional subspaces. Let us still introduce the Banach space $\mathcal{X}:=\left\{X \in \mathcal{S}^{*} ; E^{*} \mathfrak{C}^{*} X \in \mathcal{H}^{*}\right\}$. We further assume $\mathfrak{C} \in \operatorname{Lin}(\mathcal{E}, \mathcal{S})$ invertible and that the collection of the interpolation operators $\left\{I_{h}: \mathcal{S} \rightarrow S_{h}\right\}_{h>0}$ is bounded in $\operatorname{Lin}(\mathcal{S}, \mathcal{S})$.

Proposition 4.1 (Numerical stability). Let $F$ be constant in time, valued in $\mathcal{H}^{*}, G \in W^{1,1}(0, T ; \mathcal{S}), u_{0} \in \mathcal{U}$ so that $\Sigma_{0}=\mathfrak{C} E u_{0} \in \mathcal{S}, v_{0} \in \mathcal{H}, z_{0} \in \mathcal{Z}$, the functionals $\mathcal{T}$, $\Phi$, and $\Psi$ be coercive and $\Phi_{\Sigma}^{\prime}(\Sigma, \cdot)$ be Lipschitz continuous uniformly for $\Sigma \in \mathcal{S}$ in the sense

$$
\begin{array}{lr}
\exists \epsilon>0 p \geq 2 \forall(\Sigma, v, z) \in \mathcal{S} \times \mathcal{H} \times \mathcal{Z}: & \\
\mathcal{T}(v) \geq \epsilon\|v\|_{\mathcal{H}}^{2}, & \Phi(\Sigma, z) \geq \epsilon\|\Sigma\|_{\mathcal{S}}^{2}+\epsilon\|z\|_{\mathcal{Z}}^{2}, \quad \Psi(z) \geq \epsilon\|z\|_{\mathcal{Z}_{1}}^{p}, \\
\exists C \forall \Sigma \in \mathcal{S}, z \in \mathcal{Z}: & \left\|\Phi_{\Sigma}^{\prime}(\Sigma, z)\right\|_{\mathcal{S}^{*}} \leq C\left(1+\|\Sigma\|_{\mathcal{S}}+\|z\|_{\mathcal{Z}}\right), \\
\exists \ell \in \mathbb{R} \forall \Sigma \in \mathcal{S}, z, \widetilde{z} \in \mathcal{Z}: & \left\|\Phi_{\Sigma}^{\prime}(\Sigma, z)-\Phi_{\Sigma}^{\prime}(\Sigma, \widetilde{z})\right\|_{\mathcal{S}^{*}} \leq \ell\|z-\widetilde{z}\|_{\mathcal{Z}_{1}} .
\end{array}
$$

Let also the CFL condition (4.1) hold with $\tau>0$ sufficiently small (in order to make the discrete Gronwall inequality effective). 
Then the following a priori estimates hold:

$$
\begin{aligned}
& \left\|u_{\tau h}\right\|_{W^{1, \infty}(0, T ; \mathcal{H})} \leq C, \\
& \left\|\Sigma_{\tau h}\right\|_{L^{\infty}(0, T ; \mathcal{S})} \leq C \quad \text { and } \quad\left\|\dot{\Sigma}_{\tau h}\right\|_{L^{1}\left(0, T ; \mathcal{X}^{*}\right)} \leq C, \\
& \left\|v_{\tau h}\right\|_{L^{\infty}(0, T ; \mathcal{H})} \leq C \quad \text { and } \quad\left\|\mathcal{T}^{\prime} \dot{v}_{\tau h}\right\|_{L^{\infty}\left(0, T ; \mathcal{U}^{*}\right)} \leq C, \\
& \left\|z_{\tau h}\right\|_{L^{\infty}(0, T ; \mathcal{Z})} \leq C \quad \text { and } \quad\left\|\dot{z}_{\tau h}\right\|_{L^{p}\left(0, T ; \mathcal{Z}_{1}\right)} \leq C .
\end{aligned}
$$

Proof. The energy imbalance that we have here is (3.7) which can be re-written as

$$
\begin{aligned}
\frac{\mathfrak{E}_{h}^{k+1}-\mathfrak{E}_{h}^{k}}{\tau}+\Xi\left(\frac{z_{\tau h}^{k+1}-z_{\tau h}^{k}}{\tau}\right) \leq & \left\langle F_{\tau h}^{k}, v_{\tau h}^{k}\right\rangle_{\mathcal{H}^{*} \times \mathcal{H}}+\left\langle\frac{\Phi_{\Sigma}^{\prime}\left(\sum_{\tau h}^{k+1}, z_{\tau h}^{k+1}\right)+\Phi_{\Sigma}^{\prime}\left(\sum_{\tau h}^{k}, z_{\tau h}^{k}\right)}{2}, D_{\tau h}^{k}\right\rangle_{\mathcal{S}^{*} \times \mathcal{S}} \\
& -\frac{\tau}{2}\left\langle\frac{\Phi_{\Sigma}^{\prime}\left(\Sigma_{\tau h}^{k+1}, z_{\tau h}^{k+1}\right)-\Phi_{\Sigma}^{\prime}\left(\sum_{\tau h}^{k+1}, z_{\tau h}^{k}\right)}{\tau}, \frac{\sum_{\tau h}^{k+1}-\Sigma_{\tau h}^{k}}{\tau}\right\rangle_{\mathcal{S}^{*} \times \mathcal{S}}
\end{aligned}
$$

with an analog of the energy (1.9), namely

$$
\mathfrak{E}_{h}^{k+1}=\frac{1}{2}\left\langle\mathcal{T}^{\prime} v_{\tau h}^{k+1}, v_{\tau h}^{k}\right\rangle_{\mathcal{H}^{*} \times \mathcal{H}}+\Phi\left(\sum_{\tau h}^{k+1}, z_{\tau h}^{k+1}\right)
$$

We need to show that $\mathfrak{E}_{h}^{k+1}$ is indeed a sum of the kinetic and the stored energies at least up to some positive coefficients. To do so, like e.g. Lemma 4.2 of [45] or Section 6.1.6 of [50], let us write

$$
\begin{aligned}
\left\langle\mathcal{T}^{\prime} v_{\tau h}^{k+1}, v_{\tau h}^{k}\right\rangle= & \left\langle\mathcal{T}^{\prime} \frac{v_{\tau h}^{k+1}+v_{\tau h}^{k}}{2}, \frac{v_{\tau h}^{k+1}+v_{\tau h}^{k}}{2}\right\rangle-\left\langle\mathcal{T}^{\prime} \frac{v_{\tau h}^{k+1}-v_{\tau h}^{k}}{2}, \frac{v_{\tau h}^{k+1}-v_{\tau h}^{k}}{2}\right\rangle \\
= & \left\langle\mathcal{T}^{\prime} \frac{v_{\tau h}^{k+1}+v_{\tau h}^{k}}{2}, \frac{v_{\tau h}^{k+1}+v_{\tau h}^{k}}{2}\right\rangle \\
& -\frac{\tau^{2}}{4}\left\langle E_{h}^{*}\left(\mathfrak{C}^{*} I_{h}^{*} \Phi_{\Sigma}^{\prime}\left(\sum_{\tau h}^{k+1}, z_{\tau h}^{k+1}\right)-F_{\tau h}^{k+1 / 2}\right),\left(\mathcal{T}^{\prime}\right)^{-1} E_{h}^{*}\left(\mathfrak{C}^{*} I_{h}^{*} \Phi_{\Sigma}^{\prime}\left(\Sigma_{\tau h}^{k+1}, z_{\tau h}^{k+1}\right)-F_{\tau h}^{k+1 / 2}\right)\right\rangle,
\end{aligned}
$$

where all the duality pairings are between $\mathcal{H}^{*}$ and $\mathcal{H}$; here also (3.1c) has been used. Thus, using also $\mathcal{T}(v)=$ $\frac{1}{2}\left\langle\mathcal{T}^{\prime} v, v\right\rangle$, we can write the energy (4.5) as

$$
\begin{gathered}
\mathfrak{E}_{h}^{k+1}=\mathcal{T}\left(v_{\tau h}^{k+1 / 2}\right)+a_{\tau h}^{k+1} \Phi\left(\sum_{\tau h}^{k+1}, z_{\tau h}^{k+1}\right)+\frac{\tau^{2}}{2}\left\langle\left(\mathcal{T}^{\prime}\right)^{-1} E_{h}^{*} \mathfrak{C}^{*} I_{h}^{*} \Phi_{\Sigma}^{\prime}\left(\sum_{\tau h}^{k+1}, z_{\tau h}^{k+1}\right), F_{\tau h}^{k+1 / 2}\right\rangle-\frac{\tau^{2}}{4}\left\|F_{\tau h}^{k+1 / 2}\right\|_{\mathcal{H}}^{2} \\
\quad \text { with } \quad a_{\tau h}^{k+1}:=1-\frac{\tau^{2}}{4} \frac{\left\langle E_{h}^{*} \mathfrak{C}^{*} I_{h}^{*} \Phi_{\Sigma}^{\prime}\left(\sum_{\tau h}^{k+1}, z_{\tau h}^{k+1}\right),\left(\mathcal{T}^{\prime}\right)^{-1} E_{h}^{*} \mathfrak{C}^{*} I_{h}^{*} \Phi_{\Sigma}^{\prime}\left(\Sigma_{\tau h}^{k+1}, z_{\tau h}^{k+1}\right)\right\rangle}{\Phi\left(\Sigma_{\tau h}^{k+1}, z_{\tau h}^{k+1}\right)} \geq \eta
\end{gathered}
$$

and with $v_{\tau h}^{k+1 / 2}:=\frac{1}{2} v_{\tau h}^{k+1}+\frac{1}{2} v_{\tau h}^{k}$. The energy $\mathfrak{E}_{h}^{k+1}$ yields a priori estimates if the coefficient $a_{\tau h}^{k}$ is non-negative, which is just ensured by our CFL condition (4.1) used for $\Sigma_{h}=\Sigma_{\tau h}^{k+1}$ and $z_{h}=z_{\tau h}^{k+1}$. Here $\eta>0$ in (4.7) is just from (4.1). 
Altogether, summing (4.4) for $k=0, \ldots, l \leq T / \tau-1$ and using (4.7), we obtain the estimate

$$
\begin{aligned}
\epsilon & \left.\left\|v_{\tau h}^{l+1 / 2}\right\|_{\mathcal{H}}^{2}+a_{\tau h}^{l+1}\left\|\Sigma_{\tau h}^{l+1}\right\|_{\mathcal{S}}^{2}+a_{\tau h}^{l+1}\left\|z_{\tau h}^{l+1}\right\|_{\mathcal{Z}}^{2}+\tau \sum_{k=0}^{l}\left\|\frac{z_{\tau h}^{k+1}-z_{\tau h}^{k}}{\tau}\right\|_{\mathcal{Z}_{1}}^{p}\right) \\
\leq & \frac{\tau^{2}}{4}\left\|F_{\tau h}^{l+1 / 2}\right\|_{\mathcal{H}}^{2}-\frac{\tau^{2}}{2}\left\langle\left(\mathcal{T}^{\prime}\right)^{-1} E_{h}^{*} \mathfrak{C}^{*} I_{h}^{*} \Phi_{\Sigma}^{\prime}\left(\Sigma_{\tau h}^{l+1}, z_{\tau h}^{l+1}\right), F_{\tau h}^{l+1 / 2}\right\rangle-\frac{\tau^{2}}{2}\left\langle\left(\mathcal{T}^{\prime}\right)^{-1} E_{h}^{*} \mathfrak{C}^{*} I_{h}^{*} \Phi_{\Sigma}^{\prime}\left(\Sigma_{\tau h}^{0}, z_{\tau h}^{0}\right), F_{\tau h}^{1 / 2}\right\rangle \\
& +\mathcal{T}\left(v_{\tau h}^{-1 / 2}\right)+a_{\tau h}^{0} \Phi\left(\Sigma_{\tau h}^{0}, z_{\tau h}^{0}\right)+\tau \sum_{k=0}^{l}\left(\left\langle F_{\tau h}^{k+1 / 2}, v_{\tau h}^{k}\right\rangle\right. \\
& \left.+\frac{1}{2}\left\|\Phi_{\Sigma}^{\prime}\left(\Sigma_{\tau h}^{k+1}, z_{\tau h}^{k+1}\right)+\Phi_{\Sigma}^{\prime}\left(\sum_{\tau h}^{k}, z_{\tau h}^{k}\right)\right\|_{\mathcal{S}^{*}}\left\|D_{\tau h}^{k}\right\|_{\mathcal{S}}+\frac{\tau}{2} \ell\left\|\frac{z_{\tau h}^{k+1}-z_{\tau h}^{k}}{\tau}\right\|_{\mathcal{Z}_{1}}\left\|\frac{\Sigma_{\tau h}^{k+1}-\Sigma_{\tau h}^{k}}{\tau}\right\|_{\mathcal{S}}\right)
\end{aligned}
$$

where $\epsilon, p, \ell$ and $a_{\tau h}^{l+1}$ come from (4.2) and (4.7). Here we also have used that the collection $\left\{I_{h}\right\}_{h>0}$ is bounded. Using (4.2b), we estimate $\left\|\Phi_{\Sigma}^{\prime}\left(\sum_{\tau h}^{k}, z_{\tau h}^{k}\right)\right\|_{\mathcal{S}^{*}} \leq C\left(1+\left\|\Sigma_{\tau h}^{k}\right\|_{\mathcal{S}}^{2}+\left\|z_{\tau h}^{k}\right\|_{\mathcal{Z}}^{2}\right)$ and $\left\|\Phi_{\Sigma}^{\prime}\left(\Sigma_{\tau h}^{k+1}, z_{\tau h}^{k+1}\right)\right\|_{\mathcal{S}^{*}} \leq$ $C\left(1+\left\|\Sigma_{\tau h}^{k+1}\right\|_{\mathcal{S}}^{2}+\left\|z_{\tau h}^{k+1}\right\|_{\mathcal{Z}}^{2}\right)$, and then use the summability of $\left\|D_{\tau h}^{k}\right\|_{\mathcal{S}}$ needed for the discrete Gronwall inequality; here the assumption $\dot{G} \in L^{1}(0, T ; \mathcal{S})$ is needed. The last term in (4.8) is to be estimated by the Young inequality as

$$
\begin{aligned}
\frac{\tau}{2} \ell\left\|\frac{z_{\tau h}^{k+1}-z_{\tau h}^{k}}{\tau}\right\|_{\mathcal{Z}_{1}}\left\|\frac{\sum_{\tau h}^{k+1}-\Sigma_{\tau h}^{k}}{\tau}\right\|_{\mathcal{S}} & \leq \frac{\epsilon}{2}\left\|\frac{z_{\tau h}^{k+1}-z_{\tau h}^{k}}{\tau}\right\|_{\mathcal{Z}_{1}}^{2}+\frac{\ell^{2}}{8 \epsilon}\left\|\Sigma_{\tau h}^{k+1}-\Sigma_{\tau h}^{k}\right\|_{\mathcal{S}}^{2} \\
& \leq \frac{\epsilon}{2}\left\|\frac{z_{\tau h}^{k+1}-z_{\tau h}^{k}}{\tau}\right\|_{\mathcal{Z}_{1}}^{p}+C_{p, \epsilon, \ell}\left(1+\left\|\Sigma_{\tau h}^{k+1}\right\|_{\mathcal{S}}^{2}+\left\|\Sigma_{\tau h}^{k}\right\|_{\mathcal{S}}^{2}\right)
\end{aligned}
$$

with some $C_{p, \epsilon, \ell}$ depending on $p, \epsilon$, and $\ell$. Here we needed $p \geq 2$; note that this is related with the specific explicit time discretization due to the last term in (3.7) but not with the problem itself. Then we use the discrete Gronwall inequality to obtain the former estimates in (4.3b), (4.3c) and the estimates (4.3a), (4.3d). Using the discrete Gronwall inequality is a bit tricky because of the term $\left\|v_{\tau h}^{l+1 / 2}\right\|_{\mathcal{H}}^{2}$ on the left-hand side of (4.8) while there is $v_{\tau h}^{k}$ instead of $v_{\tau h}^{k+1 / 2}$ on the right-hand side of (4.8). To cope with it, we have to rely on $F$ being constant (as assumed). We prove the estimate for $l=1$, then we sum up (4.8) for $l+1$ and $l$ to get $\left\langle F_{\tau h}^{k+1 / 2}, v_{\tau h}^{k+1 / 2}\right\rangle$ also on the right-hand side. Note also that, in view of (3.6) for $k=0$, we have obtained the term $\mathcal{T}\left(v_{\tau h}^{-1 / 2}\right)$ on the right-hand side of (4.8) which, however, can simply be ignored if taking the "fictitious" velocity at level $k=-1$ as $-v_{\tau h}^{0}=-v_{h}^{0}$.

The equation $\dot{\Sigma}_{\tau h}=I_{h} \mathfrak{C} E_{h} \bar{v}_{\tau h}+\dot{G}_{\tau h}$ gives the latter estimate in (4.3b) by estimating

$$
\begin{aligned}
\int_{0}^{T}\left\langle\dot{\Sigma}_{\tau h}, X\right\rangle_{\mathcal{X}^{*} \times \mathcal{X}} \mathrm{d} t & =\int_{0}^{T}\left\langle I_{h} \mathfrak{C} E_{h} \bar{v}_{\tau h}+\dot{G}_{\tau h}, X\right\rangle_{\mathcal{X}^{*} \times \mathcal{X}} \mathrm{d} t \\
& =\int_{0}^{T}\left\langle\bar{v}_{\tau h}, E_{h}^{*} \mathfrak{C}^{*} I_{h}^{*} X\right\rangle_{\mathcal{H} \times \mathcal{H}^{*}} \mathrm{~d} t+\int_{0}^{T}\left\langle\dot{G}_{\tau h}, X\right\rangle_{\mathcal{X}^{*} \times \mathcal{X}} \mathrm{d} t
\end{aligned}
$$

for $X \in L^{\infty}(0, T ; \mathcal{X})$ and using also the already proved boundedness of $\bar{v}_{\tau h}$ in $L^{\infty}(0, T ; \mathcal{H})$ and the assumed boundedness of $E_{h}$ uniform in $h>0$; here we used also that $\dot{\Sigma}_{\tau h}(t) \in \mathcal{S} \subset \mathcal{X}^{*}$.

Eventually, the already obtained estimates $(4.2 \mathrm{~b})$ give $\Phi_{\Sigma}^{\prime}\left(\bar{\Sigma}_{\tau h}, \bar{z}_{\tau h}\right)$ bounded in $L^{\infty}\left(0, T ; \mathcal{S}^{*}\right)$. Therefore $\bar{S}_{\tau h}=\mathfrak{C}^{*} I_{h}^{*} \Phi_{\Sigma}^{\prime}\left(\bar{\Sigma}_{\tau h}, \bar{z}_{\tau h}\right)$ is bounded in $L^{\infty}\left(0, T ; \mathcal{E}^{*}\right)$, hence $E_{h}^{*} \bar{S}_{\tau h}$ is bounded in $L^{\infty}\left(0, T ; \mathcal{U}^{*}\right)$, so that $\mathcal{T}^{\prime} \dot{v}_{\tau h}=$ $\bar{F}_{\tau h}-E_{h}^{*} \bar{S}_{\tau h}$ gives the latter estimate in $(4.3 \mathrm{c})$.

We will now need the approximation properties for $h \rightarrow 0$ :

$$
\begin{aligned}
& v \in \mathcal{U}, \quad v_{h} \in V_{h}, \quad v_{h} \rightarrow v \quad \text { in } \mathcal{H} \Rightarrow E_{h} v_{h} \rightarrow E v \quad \text { in } \mathcal{E} \quad \text { with } E \in \operatorname{Lin}(\mathcal{U}, \mathcal{E}), \\
& \Sigma \in \mathcal{S}, \Sigma_{h} \in S_{h}, \Sigma_{h} \rightarrow \Sigma \text { in } \mathcal{S} \Rightarrow I_{h} \Sigma_{h} \rightarrow \Sigma \quad \text { in } \mathcal{S} .
\end{aligned}
$$


Proposition 4.2 (Convergence). Let (2.7) and (4.10a) hold, all the involved Banach spaces be separable, and the assumptions of Proposition 4.1 hold. Moreover, let

$$
\begin{aligned}
& \forall z \in \mathcal{Z}: \Phi_{\Sigma}^{\prime}(\cdot, z) \text { continuous linear, and } \Phi_{\Sigma}^{\prime}: \mathcal{S} \times \mathcal{Z}_{0} \rightarrow \operatorname{Lin}\left(\mathcal{S}, \mathcal{S}^{*}\right) \text { continuous } \\
& \quad \text { or } \Phi_{\Sigma}^{\prime}: \mathcal{S} \times \mathcal{Z} \rightarrow \mathcal{S}^{*} \text { is continuous linear, } \\
& \forall z \in \mathcal{Z}:\left[\Phi_{0}\right]_{z}^{\prime}(\cdot, z) \text { continuous linear, and }\left[\Phi_{0}\right]_{z}^{\prime}: \mathcal{S} \times \mathcal{Z}_{0} \rightarrow \operatorname{Lin}\left(\mathcal{Z}_{0}, \mathcal{Z}_{1}\right) \text { continuous } \\
& \quad \text { or }\left[\Phi_{0}\right]_{z}^{\prime}: \mathcal{S} \times \mathcal{Z} \rightarrow \mathcal{Z}_{1}^{*} \text { is continuous linear, and } \\
& \Phi_{1}^{\prime}: \mathcal{Z} \rightarrow \mathcal{Z}^{*} \text { is linear continuous, }
\end{aligned}
$$

for some Banach space $\mathcal{Z}_{0}$ into which $\mathcal{Z}$ is embedded compactly, where $\Phi_{0}$ and $\Phi_{1}$ are from (2.7). Then there is a selected subsequence, again denoted $\left\{\left(u_{\tau h}, \Sigma_{\tau h}, v_{\tau h}, z_{\tau h}\right)\right\}_{\tau>0}$ converging weakly* in the topologies indicated in the estimates (4.3) to some $(u, \Sigma, v, z)$. Moreover, any $(u, \Sigma, v, z)$ obtained as such a limit is a weak solution according Definition 2.1.

Proof. By the Banach selection principle, we can select the weakly* converging subsequence as claimed; here the separability of the involved Banach spaces is used.

Referring to the compact embedding $\mathcal{Z} \subset \mathcal{Z}_{0}$ used in the former option in (4.11a), (4.11b) and relying on a generalization the Aubin-Lions compact-embedding theorem with $\dot{\bar{z}}_{\tau h}$ being bounded in the space of the $\mathcal{Z}_{1}$-valued measures on $I$, cf. ([39], Cor. 7.9), we have $\bar{z}_{\tau h} \rightarrow z$ strongly in $L^{r}\left(0, T ; \mathcal{Z}_{1}\right)$ for any $1 \leq r<+\infty$.

Further, we realize that the approximate solution satisfy identities/inequality analogous to what is used in Definition 2.1. In view of (2.8a), the equations (3.9c) now means

$$
\int_{0}^{T}\left\langle\Phi_{\Sigma}^{\prime}\left(\bar{\Sigma}_{\tau h}, \bar{z}_{\tau h}\right), I_{h} \mathfrak{C} E_{h} \widetilde{v}\right\rangle_{\mathcal{S}^{*} \times \mathcal{S}}-\left\langle\mathcal{T}^{\prime} v_{\tau h}, \dot{\widetilde{v}}\right\rangle_{\mathcal{H}^{*} \times \mathcal{H}} \mathrm{d} t=\left\langle\mathcal{T}^{\prime} v_{0}, \widetilde{v}(0)\right\rangle_{\mathcal{H}^{*} \times \mathcal{H}}+\int_{0}^{T}\left\langle F_{h}, \widetilde{v}\right\rangle_{\mathcal{H}^{*} \times \mathcal{H}} \mathrm{d} t
$$

for any $\widetilde{v} \in C^{1}(0, T ; \mathcal{H})$ valued in $V_{h}$ and with $\widetilde{v}(T)=0$. Like in $(2.8 \mathrm{~b})$, the inclusion $(3.9 \mathrm{~b})$ means

$$
\begin{aligned}
\int_{0}^{T} \Psi(\widetilde{z})+\left\langle\left[\Phi_{0}\right]_{z}^{\prime}\left(\bar{\Sigma}_{\tau h}, \bar{z}_{\tau h}\right), \widetilde{z}-\dot{z}_{\tau h}\right\rangle_{\mathcal{Z}_{1}^{*} \times \mathcal{Z}_{1}} & +\left\langle\Phi_{1}^{\prime} \bar{z}_{\tau h}, \widetilde{z}\right\rangle_{\mathcal{Z}^{*} \times \mathcal{Z}} \mathrm{d} t+\Phi_{1}\left(z_{0}\right) \\
& \geq \Phi_{1}\left(z_{\tau h}(T)\right)+\int_{0}^{T} \Psi\left(\dot{z}_{\tau h}\right) \mathrm{d} t
\end{aligned}
$$

for all $\widetilde{z} \in L^{1}(0, T ; \mathcal{Z})$. This is completed by $(3.9 \mathrm{a})$.

It is further important that the equations in (3.9a) and the first equation in (3.9c) are linear, so that the weak convergence is sufficient for the limit passage there. In particular, we use (4.10a) and the Lebesgue dominatedconvergence theorem.

As to the weak convergence of (3.9a) integrated in time towards (3.1a) integrated in time, i.e. towards $\Sigma=\mathfrak{C} E u+G$ as used in Definition 2.1, we need to prove that

$$
\int_{0}^{T}\left\langle\Sigma_{\tau h}-G_{\tau h}, X\right\rangle_{\mathcal{S} \times \mathcal{S}^{*}}-\left\langle u_{\tau h}, E_{h}^{*} \mathfrak{C}^{*} X\right\rangle_{\mathcal{H} \times \mathcal{H}^{*}} \mathrm{~d} t \rightarrow \int_{0}^{T}\langle\Sigma-G, X\rangle_{\mathcal{S} \times \mathcal{S}^{*}}-\left\langle u, E^{*} \mathfrak{C}^{*} X\right\rangle_{\mathcal{H} \times \mathcal{H}^{*}} \mathrm{~d} t
$$

for any $X \in L^{1}\left(0, T ; \mathcal{S}^{*}\right)$. By $(4.10 a)$, we have also $E_{h}^{*} S \rightarrow E^{*} S$ in $\mathcal{H}$ for any $S \in \mathcal{E}^{*}$, in particular for $S=\mathfrak{C}^{*} X(t)$. Thus certainly $E_{h}^{*} \mathfrak{C}^{*} X \rightarrow E^{*} \mathfrak{C}^{*} X$ in $L^{1}(0, T ; \mathcal{H})$ strongly. Using the weak* convergence $u_{\tau h} \rightarrow u$ in $L^{\infty}(0, T ; \mathcal{H})$, we obtain (4.13). Moreover, in the limit $E u=\mathfrak{C}^{-1}(\Sigma-G) \in L^{\infty}(0, T ; \mathcal{E})$ so that $u \in L^{\infty}(0, T ; \mathcal{U})$.

For the limit passage in (4.12a), we also use $\Phi_{\Sigma}^{\prime}\left(\bar{\Sigma}_{\tau h}, \bar{z}_{\tau h}\right) \rightarrow \Phi_{\Sigma}^{\prime}(\Sigma, z)$ weakly* in $L^{\infty}\left(0, T ; \mathcal{S}^{*}\right)$ because $\Phi_{\Sigma}^{\prime}$ is continuous in the (weak $\times$ strong, weak)-mode, $c f$. (4.11a), and because of the mentioned strong convergence of $\bar{z}_{\tau h} \rightarrow z$.

Furthermore, we need to show the convergence $\left[\Phi_{0}\right]_{z}^{\prime}\left(\bar{\Sigma}_{\tau h}, \underline{\bar{z}}_{\tau h}\right) \rightarrow\left[\Phi_{0}\right]_{z}^{\prime}(\Sigma, z)$. For this, we use again the mentioned generalized Aubin-Lions theorem to have the strong convergence $\underline{\bar{z}}_{\tau h} \rightarrow z$ in $L^{r}\left(0, T ; \mathcal{Z}_{1}\right)$ for any 
$1 \leq r<+\infty$ and then the continuity of $\left[\Phi_{0}\right]_{z}^{\prime}$ in the (weak $\times$ strong,weak)-mode, $c f$. the former option in (4.11b). The limit passage of $(4.12 \mathrm{~b})$ towards $(2.8 \mathrm{~b})$ then uses also the weak lower semicontinuity of $\Phi_{1}$ and the weak convergence $z_{\tau h}(T) \rightarrow z(T)$ in $\mathcal{Z}$; here for this pointwise convergence in all time instants $t$ and in particular in $t=T$, we also used that we have some information about $\dot{z}_{\tau h}, c f .(4.3 \mathrm{~d})$.

So far, we have relied on the former options in (4.11a), (4.11b) and the Aubin-Lions compactness argument as far as the $z$-variable is concerned. If $\Phi$ is quadratic (as e.g. in the examples in Sects. 5.1 and 5.2 below), we can use the latter options in (4.11a), (4.11b) and simplify the above arguments, relying merely on the weak convergence $\bar{z}_{\tau h} \rightarrow z$ and $\underline{\bar{z}}_{\tau h} \rightarrow z$.

Remark 4.3 (Alternative weak formulation). Here, we used the weak formulation of (2.3c) containing the term $\left\langle\Phi_{z}^{\prime}(\Sigma, z), \dot{z}\right\rangle$ which often does not have a good meaning since $\dot{z}$ may not be regular enough in some applications. This term is thus eliminated by substituting it, after integration over the time interval, by $\Phi(\Sigma(T), z(T))-$ $\int_{0}^{T}\left\langle\Phi_{\Sigma}^{\prime}(\Sigma, z), \dot{\Sigma}\right\rangle \mathrm{d} t-\Phi\left(\Sigma_{0}, z_{0}\right)$ or even rather by $\Phi(\Sigma(T), z(T))-\int_{0}^{T}\left\langle\Phi_{\Sigma}^{\prime}(\Sigma, z), \mathfrak{C} E v\right\rangle \mathrm{d} t-\Phi\left(\Sigma_{0}, z_{0}\right)$. This however, would bring even more difficulties because we would need to prove a strong convergence of $\Phi_{\Sigma}^{\prime}(\Sigma, z)$, or of $\dot{\Sigma}$, or $\mathfrak{C} E v$ in our explicit-discretization scheme, which seems not easy.

Remark 4.4 (Nonquadratic $\Phi(\Sigma, \cdot)$ ). Some applications use such $\Phi(\Sigma, \cdot)$ which is not quadratic. This is still consistent with the explicit leap-frog-type discretization if, instead of $\Phi_{z}^{\prime}(\Sigma, z)$, we consider an abstract difference quotient $\Phi_{z}^{\circ}(\Sigma, z, \widetilde{z})$ with the properties

$$
\Phi_{z}^{\circ}(\Sigma, z, z)=\Phi_{z}^{\prime}(\Sigma, z) \quad \text { and } \quad\left\langle\Phi_{z}^{\circ}(\Sigma, z, \widetilde{z}), z-\widetilde{z}\right\rangle=\Phi(\Sigma, z)-\Phi(\Sigma, \widetilde{z})
$$

cf. [41]. Then, instead of $\Phi_{z}^{\prime}\left(\sum_{\tau h}^{k+1}, \frac{z_{\tau h}^{k+1}+z_{\tau h}^{k}}{2}\right)$ in (3.1b), we should write $\Phi_{z}^{\circ}\left(\sum_{\tau h}^{k+1}, z_{\tau h}^{k+1}, z_{\tau h}^{k}\right)$.

Remark 4.5 (State-dependent dissipation). The generalization of $\Psi$ dependent also on $z$ or even on $(\Sigma, z)$ is easy. Then $\partial \Psi$ is to be replaced by the partial subdifferential $\partial_{\dot{z}} \Psi$ and $(3.1 b)$ should use $\Psi\left(\sum_{\tau h}^{k+1}, z_{\tau h}^{k}, \cdot\right)$ instead of $\Psi(\cdot)$.

Remark 4.6 (Spatial numerical approximation). From the coercivity of the stored energy $\Phi$, we have $\Sigma_{\tau h}^{k} \in \mathcal{S}$ for any $k=0,1, \ldots$ and thus, from (3.1a), $E_{h} v_{\tau h}^{k} \in \mathcal{E}$ so that $v_{\tau h}^{k} \in \mathcal{U}$, although the limit $v$ cannot be assumed valued in $\mathcal{U}$ in general. Similarly, from (3.1c), one can read that $E_{h}^{*} S_{\tau h}^{k} \in \mathcal{H}$ although this cannot be expected in the limit in general. Anyhow, on the time-discrete level, one can use the FEM discretization similarly as in the linear elastodynamics where regularity can be employed, $c f .[7,8,11,50]$ for a mixed finite-element method and [18] for the more recently developed staggered discontinuous Galerkin method for elastodynamics.

Remark 4.7 (Other explicit-implicit schemes). Combination of explicit and implicit time discretization might not only be due to parabolic evolution of internal variables but also due to geometrical reasons, e.g. transmission through a thin layer, that lead to a very restrictive CFL condition, $c f$. [14].

\section{Particular examples}

We present three examples from continuum mechanics of deformable bodies at small strains of different characters to illustrate applicability of the ansatz (2.2) and the above discretization scheme. Various combinations of these examples are possible, too, covering thus a relatively wide variety of models.

We use a standard notation concerning function spaces. Beside the Lebesgue $L^{p}$-spaces, we denote by $H^{k}\left(\Omega ; \mathbb{R}^{n}\right)$ the Sobolev space of functions whose distributional derivatives are from $L^{2}\left(\Omega ; \mathbb{R}^{n \times d^{k}}\right)$. 


\subsection{Plasticity or creep}

The simplest example with quadratic stored energy and local dissipation potential is the model of plasticity or creep. The internal variable is then the plastic strain $\pi$, valued in the set of symmetric trace-free matrices $\mathbb{R}_{\mathrm{dev}}^{d \times d}=\left\{P \in \mathbb{R}^{d \times d} ; P^{\top}=P\right.$, tr $\left.P=0\right\}$. For simplicity, we consider only homogeneous Neumann or Dirichlet boundary conditions, so that simply $E=e(u)$ and $\mathfrak{C}=\mathbb{C}$. The stored energy in terms of strain $e(u)$ is

$$
\mathscr{W}(u, \pi)=\int_{\Omega} \frac{1}{2} \mathbb{C}(e(u)-\pi):(e(u)-\pi) \mathrm{d} x,
$$

which is actually a function of the elastic strain $e_{\mathrm{el}}=e-\pi$. The additive decomposition $e(u)=e_{\mathrm{el}}+\pi$ is referred to as Green-Naghdi's [26] decomposition. This energy leads to

$$
\Phi(\sigma, \pi)=\int_{\Omega} \frac{1}{2} \mathbb{C}^{-1} \sigma: \sigma-\sigma: \pi+\frac{1}{2} \mathbb{C} \pi: \pi \mathrm{d} x \quad \text { with } \quad \sigma=\mathbb{C} e(u) .
$$

Let us note that $\Phi_{\sigma}^{\prime}(\sigma, \pi)=\mathbb{C}^{-1} \sigma-\pi=e-\pi$, i.e. the elastic strain $e_{\mathrm{el}}$, and that the proto-stress $\Sigma=\sigma$ is indeed different from the actual stress $\sigma-\mathbb{C} \pi$.

The dissipation potential is standardly chosen as

$$
\Psi(\dot{\pi})=\int_{\Omega} \sigma_{\mathrm{Y}}|\dot{\pi}|+\frac{1}{2} \mathbb{D} \dot{\pi}: \dot{\pi} \mathrm{d} x
$$

with $\sigma_{\mathrm{Y}} \geq 0$ a prescribed yield stress and $\mathbb{D}$ a positive semidefinite viscosity tensor. The dissipation rate is then $\Xi(\dot{\pi})=\int_{\Omega} \sigma_{\mathrm{Y}}|\dot{\pi}|+\mathbb{D} \dot{\pi}: \dot{\pi} \mathrm{d} x$. For $\mathbb{D}>0$ and $\sigma_{\mathrm{Y}}=0$, we obtain mere creep model or, in other words, the linear viscoelastic model in the Maxwell rheology. For both $\mathbb{D}>0$ and $\sigma_{\mathrm{Y}}>0$, we obtain viscoplasticity. For $\mathbb{D}=0$ and $\sigma_{\mathrm{Y}}>0$, we would obtain the rate-independent (perfect) plasticity but our Proposition 4.1 does not cover this case (i.e. $p=1$ is not admitted).

The functional setting is $\mathcal{H}=L^{2}\left(\Omega ; \mathbb{R}^{d}\right), \mathcal{E}=\mathcal{S}=\mathcal{Z}=\mathcal{Z}_{1}=L^{2}\left(\Omega ; \mathbb{R}_{\text {sym }}^{d \times d}\right)$ where $\mathbb{R}_{\text {sym }}^{d \times d}$ denotes symmetric $(d \times d)$-matrices. Thus $\mathcal{U}:=\left\{v \in L^{2}\left(\Omega ; \mathbb{R}^{d}\right) ; e(v) \in L^{2}\left(\Omega ; \mathbb{R}^{d \times d}\right)\right\}=H^{1}\left(\Omega ; \mathbb{R}^{d}\right)$ by Korn's inequality.

A modification of the stored energy models an isotropic hardening, enhancing (5.1) as

$$
\mathscr{W}(u, \pi)=\int_{\Omega} \frac{1}{2} \mathbb{C}_{1}(e(u)-\pi):(e(u)-\pi)+\frac{1}{2} \mathbb{C}_{2} \pi: \pi \mathrm{d} x
$$

so that the energy $\Phi$ from (5.2) is modified as

$$
\Phi(\sigma, \pi)=\int_{\Omega} \frac{1}{2} \mathbb{C}_{1}^{-1} \sigma: \sigma-\sigma: \pi+\frac{1}{2}\left(\mathbb{C}_{1}+\mathbb{C}_{2}\right) \pi: \pi \mathrm{d} x .
$$

In the pure creep variant $\sigma_{\mathrm{Y}}=0$, this is actually the standard linear solid (in a so-called Zener form), considered together with the leap-frog time discretization in [9]. The isochoric constraint $\operatorname{tr} \pi=0$ can then be avoided, assuming that $\mathbb{C}_{2}$ is positive definite.

All these models lead to a flow rule which is localized on each element when an element-wise constant approximation of $\pi$ is used, and no large system of algebraic equations need to be solved so that the combination with the explicit discretization of the other equations leads to a very fast computational procedure.

Another modification for gradient plasticity by adding terms $\frac{1}{2} \kappa|\nabla \pi|^{2}$ into the stored energy is easily possible, too. This modification uses $\mathcal{Z}=H^{1}\left(\Omega ; \mathbb{R}_{\text {sym }}^{d \times d}\right)$ and (2.7) with $\Phi_{1}(z)=\int_{\Omega} \frac{1}{2} \kappa|\nabla \pi|^{2}$ and makes, however, the flow rule nonlocal but at least one can benefit from that the usual space discretization of the proto-stress $\sigma$ uses the continuous piecewise smooth elements which allows for handling gradients $\nabla \pi$ if used consistently also for $\pi$. For the quasistatic variant of this model, we refer to the classical monographs $[28,49]$, while the dynamical model with $\mathbb{D}=0$ is e.g. in Section 5.2 of [37]. 
Noteworthy, all these models bear time regularity if the loading is smooth and initial conditions regular enough, which can be advantageously reflected in space FEM approximation, too.

The Maxwell visco-elastodynamics was also studied by J.-P. Groby ([27], Part I, Sect. 2) using a slightly modified time discretization scheme, namely the order of (3.1a) and (3.1c) was exchanged.

The CFL condition (4.1) here is actually the same as the standard one (1.4). This is because the internal variable actually does not influence the elasticity response and, likewise, the inertia is independent of the internal variable, so the wave speed is not influenced either. The CFL is thus of the form $\tau \leq h \sqrt{\varrho /\left|\lambda_{\max }(\mathbb{C})\right|}$ where $\lambda_{\max }(\mathbb{C})$ is the maximal eigenvalue of $\mathbb{C}$.

\subsection{Poroelasticity in isotropic materials}

Another example with quadratic stored energy but less trivial dissipation potential is a saturated Darcy or Fick flow of a diffusant in porous media, e.g. water in porous elastic rocks or in concrete, or a solvent in elastic polymers. The most simple model is the classical Biot model [12], capturing effects as swelling or seepage. In a one-component flow, the internal variable is then the scalar-valued diffusant content (or concentration) denoted by $\zeta$.

As in the previous Section 5.1, we consider only Neumann or Dirichlet boundary conditions, so that $E=e(u)$. Here we use the orthogonal decomposition $e=\operatorname{sph} e+\operatorname{dev} e$ with the spherical (volumetric) part sph $e:=(\operatorname{tr} e) \mathbb{I} / d$ and the deviatoric part dev $e$ and confine ourselves to isotropic materials where the elastic-moduli tensor $\mathbb{C}_{i j k l}=$ $K \delta_{i j} \delta_{k l}+G\left(\delta_{i k} \delta_{j l}+\delta_{i l} \delta_{j k}-2 \delta_{i j} \delta_{k l} / d\right)$ with $K$ the bulk modulus and $G$ the shear modulus (=the second Lamé constant), which is the standard notation hopefully without any confusion with the notation used in (1.6). Then the proto-stress $\Sigma=\sigma=\mathbb{C} e=K \operatorname{sph} e+2 G \operatorname{dev} e$. In particular, $\operatorname{sph} \sigma=K \operatorname{sph} e$ so that $\operatorname{tr} e=K^{-1} \operatorname{tr} \sigma$.

Adopting the gradient theory for this internal variable $\zeta$, the stored energy in terms of strain is considered

$$
\begin{aligned}
\mathscr{W}(u, \zeta)= & \int_{\Omega} \frac{1}{2} \mathbb{C} e(u): e(u)+\frac{1}{2} M(\beta \operatorname{tr} e(u)-\zeta)^{2}+\frac{1}{2} L\left(\zeta-\zeta_{\mathrm{eq}}\right)^{2}+\frac{\kappa}{2}|\nabla \zeta|^{2} \mathrm{~d} x \\
= & \int_{\Omega} \frac{1}{2}\left(K+\frac{\beta^{2}}{d} M\right)|\operatorname{sph} e(u)|^{2}+G|\operatorname{dev} e(u)|^{2} \\
& -\beta M \zeta \operatorname{tr} e(u)+\frac{1}{2} M \zeta^{2}+\frac{1}{2} L\left(\zeta-\zeta_{\text {eq }}\right)^{2}+\frac{\kappa}{2}|\nabla \zeta|^{2} \mathrm{~d} x
\end{aligned}
$$

which, in terms of the (here partial) stress $\sigma=\mathbb{C} e$, reads as $\int_{\Omega} \frac{1}{2}\left(\frac{1}{K}+\frac{\beta^{2}}{d K^{2}} M\right)|\operatorname{sph} \sigma|^{2}+\frac{1}{G}|\operatorname{dev} \sigma|^{2}-\beta \zeta \frac{M}{K} \operatorname{tr} \sigma+$ $\frac{1}{2} M \zeta^{2}+\frac{1}{2} L\left(\zeta-\zeta_{\text {eq }}\right)^{2} \mathrm{~d} x$. Here $M>0$ and $\beta>0$ are so-called Biot modulus and coefficient, respectively, $\kappa>0$ is a capillarity coefficient, and $\zeta_{\text {eq }}$ is a given equilibrium content. From (5.6), we arrive at the overall stored energy as:

$$
\begin{aligned}
\Phi(\sigma, \zeta)= & \int_{\Omega} \frac{1}{2}\left(\frac{1}{K}+\frac{\beta^{2}}{d K^{2}} M\right)|\operatorname{sph} \sigma|^{2}+\frac{1}{G}|\operatorname{dev} \sigma|^{2}-\beta \zeta \frac{M}{K} \operatorname{tr} \sigma \mathrm{d} x \\
& +\underbrace{\int_{\Omega} \frac{1}{2} M \zeta^{2}+\frac{1}{2} L\left(\zeta-\zeta_{\mathrm{eq}}\right)^{2}+\frac{\kappa}{2}|\nabla \zeta|^{2} \mathrm{~d} x}_{=: \Phi_{1}(\zeta)} .
\end{aligned}
$$

Let us note that $\Phi_{\sigma}^{\prime}(\sigma, \zeta)=\mathbb{C}^{-1} \sigma+\frac{\beta M}{d K^{2}}(\beta \operatorname{sph} \sigma-\zeta K \mathbb{I})$, i.e. the elastic strain, and that the proto-stress $\Sigma=\sigma$ indeed differs from an actual stress by the spherical pressure part $\frac{\beta M}{d K}(\beta \operatorname{sph} \sigma-\zeta K \mathbb{I})$.

The driving force for the diffusion is the chemical potential $\mu=\Phi_{\zeta}^{\prime}(\sigma, \zeta)$, i.e. here

$$
\mu=(M+L) \zeta-\beta \frac{M}{K} \operatorname{tr} \sigma-L \zeta_{\mathrm{eq}}-\kappa \Delta \zeta
$$

The diffusion equation is

$$
\dot{\zeta}-\operatorname{div}(\mathbb{M} \nabla \mu)=0
$$


with $\mathbb{M}$ denoting the diffusivity tensor. The system (5.7) is called the Cahn-Hilliard equation, here combined with elasticity so that the flow of the diffusant is driven both by the gradient of concentration (Fick's law) and the gradient of the mechanical pressure (Darcy's law). The dissipation potential in terms of $\nabla \mu$, let us denote it by $R$ behind this system, is

$$
R(\mu)=\int_{\Omega} \frac{1}{2} \mathbb{M} \nabla \mu \cdot \nabla \mu \mathrm{d} x .
$$

For the analysis cf. e.g. ([35], Sect. 7.6).

One would expect the dissipation potential as a function of the rate of internal variables, as in (2.3c). In fact, the system (5.7) turns into the form (2.3c) if one takes the dissipation potential $\Psi=\Psi(\dot{\zeta})$ as

$$
\Psi(\dot{\zeta})=R^{*}(\dot{\zeta})
$$

with $R^{*}$ denoting the convex conjugate of $R$. Now, $\Psi$ is nonlocal. The functional setting is as in the previous example but now $\mathcal{Z}=H^{1}(\Omega)$ and $\mathcal{Z}_{1}=H^{1}(\Omega)^{*}$. For a discretization of the type (3.1b), see [40].

Often, the diffusivity is considered dependent on $\zeta$. Or even one can think about $\mathbb{M}=\mathbb{M}(\sigma, \zeta)$. Then the modification in Remark 4.5 is to be applied. In particular, $R(\sigma, \zeta, \mu)=\int_{\Omega} \frac{1}{2} \mathbb{M}(\sigma, \zeta) \nabla \mu \cdot \nabla \mu \mathrm{d} x$ and $\Psi(\sigma, \zeta, \dot{\zeta})=$ $[R(\sigma, \zeta, \cdot)]^{*}(\dot{\zeta})$.

For this Biot model in the dynamical variant, the reader is also referred to the books $[1,16,17,48]$ or also $[35,37]$. In any case, the diffusion involves gradients and in the implicit discretization it leads to large systems of algebraic equations, which inevitably slows down the fast explicit discretization of the mechanical part itself.

For this case also the CFL condition (4.1) is the same as the standard one and leads to a restriction of the form $\tau \leq C h / V_{\max }$ where $V_{\max }$ denotes the maximal speed with which waves propagate in the medium. We note that the pressure velocity which is the maximal speed of propagation in isotropic solids is enhanced in the Biot model. The stability analysis of the discrete scheme is quite technical and does not always lead to a practical CFL condition. A. Ezziani in his Ph.D. thesis [22] studied a discretization of Biot's model similar to (5.6) but the stability analysis of the discrete scheme is very nontrivial, $c f$. formula (7.4.11) of [22] and, as he points out, cannot be translated into a practical condition. Therefore, in practice he proposes to use $\tau \leq a_{r} h / V_{\mathrm{pf}}$ where $V_{\mathrm{pf}}$ is the speed of the fast wave and $a_{r}$ is a constant depending on the order of the particular space discretization used. The attenuation caused by diffusion causes also some dispersion of wave velocities which stay however bounded from above by a high-frequency limit, cf. also Figure 5.2.1 of [22], so the CFL condition expectedly holds uniformly like for the pure elastodynamics.

\subsection{Damage}

The simplest examples of nonconvex stored energy are models of damage. The most typical models use as an internal variable the scalar-valued bulk damage $\alpha$ having the interpretation as a phenomenological volume fraction of microcracks or microvoids manifested macroscopically as a certain weakening of the elastic response. This concept was invented by L.M. Kachanov [32] and Y.N. Rabotnov [38].

Considering gradient theories, the stored energy in terms of the strain and damage is here considered as

$$
\mathscr{W}(e, \alpha)=\int_{\Omega} \frac{1}{2} \gamma(\alpha) \mathbb{C} e: e+\phi(\alpha)+\frac{\kappa}{2}|\nabla \alpha|^{2}+\frac{\varepsilon}{2} \nabla(\mathbb{C} e): \nabla e \mathrm{~d} x,
$$

where $\phi(\cdot)$ is an energy of damage which gives rise to an activation threshold for damage evolution and may also lead to healing (if allowed). The last term is mainly to facilitate the mathematics towards convergence and existence of a weak solution in such purely elastic materials without involving any viscosity, $c f$. ([35], Sect. 7.5.3). This regularization can also control dispersion of elastic waves. More specifically, the 4th-order term resulted in the momentum-equilibrium equation from the $\varepsilon$-term in (5.10) causes an anomalous dispersion, i.e. waves with shorter wavelength propagate faster than longer wavelength ones, $c f . e . g$. ([35], Rem. 6.3.6). The $\nabla \alpha$-term also facilitates the analysis and controls the internal length-scale of damage profiles. 
Let us consider the "generalized" elasticity tensor $\mathfrak{C}=\mathbb{C}$ independent of $x$. As in the previous examples, $E u=e(u)$ and $G=0$. According (2.3a), the proto-stress $\Sigma=\mathfrak{C} E u+G$, denoted by $\sigma$, now looks as $\mathbb{C} e=: \sigma$; in damage mechanics, the proto-stress is also called an effective stress with a specific mechanical interpretation, cf. [38]. In terms of $\sigma$, the stored energy is then

$$
\Phi(\sigma, \alpha)=\int_{\Omega} \frac{1}{2} \gamma(\alpha) \mathbb{C}^{-1} \sigma: \sigma+\frac{\varepsilon}{2} \nabla \mathbb{C}^{-1} \sigma: \nabla \sigma \mathrm{d} x+\underbrace{\int_{\Omega} \phi(\alpha)+\frac{\kappa}{2}|\nabla \alpha|^{2} \mathrm{~d} x}_{=: \Phi_{1}(\alpha)} .
$$

Then $\Phi_{\sigma}^{\prime}=\gamma(\alpha) \mathbb{C}^{-1} \sigma-\operatorname{div}\left(\varepsilon \nabla\left(\mathbb{C}^{-1} \sigma\right)\right)$ and the true stress $S=\mathbb{C}^{*} \Phi_{\sigma}^{\prime}$ is then $\gamma(\alpha) \sigma-\operatorname{div}(\varepsilon \nabla \sigma)$ provided $\mathbb{C}$ is constant and symmetric. The damage driving force (energy) is $\Phi_{\alpha}^{\prime}(\sigma, \alpha)=\frac{1}{2} \gamma^{\prime}(\alpha) \mathbb{C}^{-1} \sigma: \sigma+\phi^{\prime}(\alpha)-\operatorname{div}(\kappa \nabla \alpha)$. When $\gamma^{\prime}(0)=0$ and $\phi^{\prime}(0) \leq 0$, then always $\alpha \geq 0$ also in the discrete scheme if $\alpha_{0} \geq 0$.

The other ingredient is the dissipation potential. To comply with the coercivity on $\mathcal{Z}_{1}=L^{2}(\Omega)$ with $p \geq 2$ as needed in Proposition 4.1, one can consider either

$$
\Psi(\dot{\alpha})=\left\{\begin{array} { c l } 
{ \int _ { \Omega } \varepsilon _ { 1 } \dot { \alpha } ^ { 2 } \mathrm { d } x } \\
{ + \infty }
\end{array} \quad \text { or } \quad \left\{\begin{array}{ll}
\int_{\Omega} \varepsilon_{1} \dot{\alpha}^{2} \mathrm{~d} x & \text { if } \dot{\alpha} \leq 0 \text { a.e. on } \Omega \\
\int_{\Omega} \dot{\alpha}^{2} / \varepsilon_{1} \mathrm{~d} x & \text { otherwise }
\end{array}\right.\right.
$$

with some (presumably small) coefficient $\varepsilon_{1}>0$. The former option corresponds to a unidirectional (i.e. irreversible) damage not allowing any healing (as used in engineering) while the latter option allows for (presumably slow) healing as used in geophysical models on large time scales.

Since $\sigma$ appears nonlinearly in $\Phi_{\alpha}^{\prime}(\sigma, \alpha)$, the strong convergence $\bar{\sigma}_{\tau h} \rightarrow \sigma$ in $L^{2}\left(Q ; \mathbb{R}^{d \times d}\right)$ is needed. For this, the strain-gradient term with $\varepsilon>0$ is needed and the Aubin-Lions compact embedding theorem is used. This gives the strong convergence even in the norm of $L^{1 / \epsilon}\left(0, T ; L^{2 d /(d-2)-\epsilon}\left(\Omega ; \mathbb{R}^{d \times d}\right)\right)$ for arbitrarily small $\epsilon>0$ provided also $\dot{\sigma}_{\tau h}$ is bounded in some norm, which can be shown by using $\dot{\sigma}_{\tau h}=\mathbb{C} e\left(\bar{v}_{\tau h}\right)$ and the Green formula

$$
\begin{aligned}
\left\|\dot{\sigma}_{\tau h}\right\|_{L^{\infty}\left(0, T ; H^{-1}\left(\Omega ; \mathbb{R}^{d \times d}\right)\right)} & =\sup _{\|\widetilde{e}\|_{L^{1}\left(0, T ; H_{0}^{1}\left(\Omega ; \mathbb{R}^{d \times d)}\right)\right.} \leq 1} \int_{0}^{T} \int_{\Omega} \dot{\sigma}_{\tau h}: \widetilde{e} \mathrm{~d} x \mathrm{~d} t \\
& =\sup _{\|\widetilde{e}\|_{L^{1}\left(0, T ; H_{0}^{1}\left(\Omega ; \mathbb{R}^{d \times d)}\right)\right.} \leq 1} \int_{0}^{T} \int_{\Omega} \mathbb{C} e\left(\bar{v}_{\tau h}\right): \widetilde{e} \mathrm{~d} x \mathrm{~d} t \\
& =\sup _{\|\widetilde{e}\|_{L^{1}\left(0, T ; H_{0}^{1}\left(\Omega ; \mathbb{R}^{d \times d)}\right)\right.} \leq 1}-\int_{0}^{T} \int_{\Omega} \bar{v}_{\tau h} \cdot \operatorname{div}(\mathbb{C} \widetilde{e}) \mathrm{d} x \mathrm{~d} t \leq C\left\|\bar{v}_{\tau h}\right\|_{L^{\infty}\left(0, T ; L^{2}\left(\Omega ; \mathbb{R}^{d}\right)\right)}
\end{aligned}
$$

with $C$ depending on $|\mathbb{C}|$. $C f$. also the abstract estimation (4.9).

When $\gamma$ or $\phi$ are not quadratic but continuously differentiable, one can use the abstract difference quotient (4.14) defined, in the classical form, as

$$
\Phi_{z}^{\circ}(\Sigma, \alpha, \widetilde{\alpha})= \begin{cases}\frac{1}{2} \frac{\gamma(\alpha)-\gamma(\widetilde{\alpha})}{\alpha-\widetilde{\alpha}} \mathbb{C}^{-1} \sigma: \sigma+\frac{\phi(\alpha)-\phi(\widetilde{\alpha})}{\alpha-\widetilde{\alpha}}-\kappa \Delta \frac{\alpha+\widetilde{\alpha}}{2} & \text { where } \alpha \neq \widetilde{\alpha}, \\ \frac{1}{2} \gamma^{\prime}(\alpha) \mathbb{C}^{-1} \sigma: \sigma+\phi^{\prime}(\alpha)-\kappa \Delta \alpha & \text { where } \alpha=\widetilde{\alpha} .\end{cases}
$$

Of course, rigorously, the $\Delta$-operator in (5.13) is to be understood in the weak form when using it in (3.1b).

Due to the gradient $\kappa$-term in (5.11), the implicit incremental problem (3.1b) leads to an algebraic problem with a full matrix, which may substantially slow down the otherwise fast explicit scheme. Like in the previous model the capillarity, now this gradient theory controls the length-scale of the damage profile and also serves as a regularization to facilitate mathematical analysis. Sometimes, a nonlocal "fractional" gradient can facilitate the analysis, too. Then, some wavelet equivalent norm can be considered to accelerate the calculations, $c f$. also [3]. As far as the stress-gradient term, it is important that the discretization of the proto-stress in the usual 
implementation of the leap-frog method is continuous piecewise smooth, so that $\nabla \sigma$ has a good sense in the discretization without need to use higher-order elements. Here we use that the latter relation in (3.1c) is to be understood in the weak form, namely $\int_{\Omega} S_{\tau h}^{k+1}: \tilde{E}_{h} \mathrm{~d} x=\left\langle\Phi_{\Sigma}^{\prime}\left(\sum_{\tau h}^{k+1}, z_{\tau h}^{k+1}\right), \mathfrak{C} \tilde{E}_{h}\right\rangle$ for $\tilde{E}_{h}=\tilde{e}_{h}=e\left(\tilde{u}_{h}\right)$, which means

$$
\int_{\Omega} S_{\tau h}^{k+1}: \tilde{E}_{h} \mathrm{~d} x=\int_{\Omega} \gamma\left(\alpha_{\tau h}^{k+1}\right) \mathbb{C}^{-1} \sigma^{k+1}: \mathbb{C} \tilde{e}_{h}+\epsilon \nabla \mathbb{C}^{-1} \sigma^{k+1}: \nabla \mathbb{C} \tilde{e}_{h} \mathrm{~d} x
$$

for any $\tilde{e}_{h}$ from the corresponding finite-dimensional subspace of $H^{1}\left(\Omega ; \mathbb{R}_{\mathrm{sym}}^{d \times d}\right)$. Thus we indeed do not need higher-order elements, and also we do not need to specify explicitly homogeneous boundary conditions in this boundary-value problem.

The functional setting is $\mathcal{H}=L^{2}\left(\Omega ; \mathbb{R}^{d}\right), \mathcal{E}=\mathcal{S}=H^{1}\left(\Omega ; \mathbb{R}_{\text {sym }}^{d \times d}\right), \mathcal{Z}=H^{1}(\Omega)$, and $\mathcal{Z}_{0}=\mathcal{Z}_{1}=L^{2}(\Omega)$. Then $\mathcal{U}=H^{2}\left(\Omega ; \mathbb{R}^{d}\right)$, and $E=e(\cdot)$ is understood as an operator $H^{2}\left(\Omega ; \mathbb{R}^{d}\right) \rightarrow H^{1}\left(\Omega ; \mathbb{R}_{\mathrm{sym}}^{d \times d}\right)$, and $\mathfrak{C}^{*} \cong \mathbb{C}^{\top}=\mathbb{C}$ is understood as an a operator from $H^{1}\left(\Omega ; \mathbb{R}_{\text {sym }}^{d \times d}\right)$ to itself.

A particular case of this model is a so-called phase-field fracture, taking as a basic choice

$$
\gamma(\alpha):=\varepsilon^{2} / \varepsilon_{0}^{2}+\alpha^{2}, \quad \phi(\alpha):=\mathfrak{g}_{\mathrm{c}}(1-\alpha)^{2} / \varepsilon, \quad \text { and } \quad \kappa:=\varepsilon \mathfrak{g}_{\mathrm{c}}
$$

with $\mathfrak{g}_{\mathrm{c}}$ denoting the energy of fracture and with $\varepsilon$ controlling a "characteristic" width of the phase-field fracture. The physical dimension of $\varepsilon_{0}$ as well as of $\varepsilon$ is $\mathrm{m}$ (meters) while the physical dimension of $\mathfrak{g}_{\mathrm{c}}$ is $\mathrm{J} / \mathrm{m}^{2}$. This is known as the so-called Ambrosio-Tortorelli functional [2]. In the dynamical context, only various implicit discretization schemes seems to be used so far, $c f .[15,29,42,46]$. There are a lot of improvements of this basic model, approximating a mode-sensitive fracture, or $\varepsilon$-insensitive models (with $\varepsilon$ referring to (5.14)), or ductile fracture, $c f$. [40]. This last variant combines this model with the plasticity as in Section 5.1.

As mentioned above in this case we have anomalous dispersion, i.e. the high frequencies propagate faster, $c f$. e.g. ([35], Rem. 6.3.6). The resulting CFL condition is a combination of the usual CFL (1.4) for the 2nd-order elastodynamic model with the CFL condition for 4th-order plate as in [10]. More specifically, the speed of elastic waves in such combined model is like $v \sim v_{0} \sqrt{1+\varepsilon / \lambda^{2}}$ with $v_{0}$ the speed in the elastodynamic case (i.e. $\varepsilon=0$ ) and with $\lambda$ the wavelength, $c f$. ([35], Rem. 6.3.6) for a one-dimensional analysis. For particular space discretisations, implementable wavelengths $\lambda$ are bounded from below just by $h$. This yields to a CFL condition of the type

$$
\tau \leq C \frac{h}{\sqrt{1+\varepsilon / h^{2}}}
$$

Asymptotically, for $h \rightarrow 0$ we can see that $\tau$ is to be small as $\mathscr{O}\left(\varepsilon^{-1 / 2} h^{2}\right)$. For fixed $\varepsilon>0$, this is actually very restrictive like in the explicit discretization of the heat equation where it practically prevents from efficient usage of explicit discretizations. However, here the role of $\varepsilon$ is primarily to facilitate rigorous existence of weak solutions of this model and can be assumed to be small. Then the influence of this 4th-order term and this restrictive asymptotics is presumably small, and the usual CFL condition resulting from (5.15) with $\varepsilon=0$ will dominate except on very fine space discretizations.

Let us eventually remark that better asymptotics of the type $\tau \sim \mathscr{O}\left(\varepsilon^{-1 / 2} h^{1+\delta}\right)$ for $h \rightarrow 0$ can be obtained by replacing the 4 th-order term by a nonlocal term of the order $2(1+\delta)$ for some $\delta>0$ small, which would even allow for more general dispersion [30] and simultaneously make the analytically desired regularization of the damage model, $c f$. . ([35], Rems. 6.3.7 and 7.5.29).

Acknowledgements. The authors are deeply thankful to Christos G. Panagiotopoulos for fruitful discussions. Also the careful and critical reading of the original version and valuable suggestions by two anonymous referees are acknowledged. This research has been partly supported from the grant 19-04956S of the Czech Sci. Foundation, and by the institutional support RVO: 61388998 (ČR). T.R. also acknowledges the hospitality of FORTH in Heraklion, Crete. 


\section{REFERENCES}

[1] Y.N. Abousleiman, A.H.-D. Cheng and F.-J. Ulm, editors, Poromechanics III: Biot Centennial (1905-2005). Taylor \& Francis, London (2005).

[2] L. Ambrosio and V.M. Tortorelli, Approximation of functional depending on jumps via by elliptic functionals via $\Gamma$-convergence. Comm. Pure Appl. Math. 43 (1990) 999-1036.

[3] M. Arndt, M. Griebel and T. Roubíček, Modelling and numerical simulation of martensitic transformation in shape memory alloys. Continuum Mech. Thermodyn. 15 (2003) 463-485.

[4] D.N. Arnold and G. Awanou, Rectangular mixed finite elements for elasticity. Math. Models Methods Appl. Sci. 15 (2005) $1417-1429$.

[5] D.N. Arnold and R. Winther, Mixed finite elements for elasticity. Numer. Math. 92 (2002) 401-419.

[6] D.N. Arnold, G. Awanou and R. Winther, Finite elements for symmetric tensors in three dimensions. Math. Comput. 77 (2008) $1229-1251$.

[7] E. Bécache, P. Joly and C. Tsogka, Fictitious domains, mixed finite elements and perfectly matched layers for 2D elastic wave propagation. J. of Comput. Acoustics 9 (2001) 1175-1202.

[8] E. Bécache, P. Joly and C. Tsogka, A new family of mixed finite elements for the linear elastodynamic problem. SIAM J. Numer. Anal. 39 (2002) 2109-2132.

[9] E. Bécache, A. Ezziani and P. Joly, A mixed finite element approach for viscoelastic wave propagation. Comput. Geosci. 8 (2004) 255-299.

[10] E. Bécache, G. Derveaux and P. Joly, An efficient numerical method for the resolution of the Kirchhoff-Love dynamic plate equation. Numer. Meth. Partial Differ. Equ. 21 (2005) 323-348.

[11] E. Bécache, J. Rodríguez and C. Tsogka, Convergence results of the fictitious domain method for a mixed formulation of the wave equation with a Neumann boundary condition. ESAIM:M2AN 43 (2009) 377-398.

[12] M.A. Biot, General theory of three-dimensional consolidation. J. Appl. Phys. 12 (1941) 155-164.

[13] T. Bohlen, Parallel 3D viscoelastic finite difference seismic modelling. Comput. Geosci. 28 (2002) 887-899.

[14] M. Bonnet, A. Burel, M. Duruflé and P. Joly, Effective transmission conditions for thin-layer transmission problems in elastodynamics. The case of a planar layer model. ESAIM:M2AN 50 (2016) 43-75.

[15] M.J. Borden, C.V. Verhoosel, M.A. Scott, T.J.R. Hughes and C.M. Landis, A phase-field description of dynamic brittle fracture. Comput. Meth. Appl. Mech. Eng. 217-220 (2012) 77-95.

[16] J.M. Carcione, Wave Fields in Real Media, Wave Propagation in Anisotropic, Anelastic, Porous and Electromagnetic Media. Elsevier, Amsterdam (2015).

[17] A.H.-D. Cheng, Poroelasticity. Springer, Switzerland (2016).

[18] E.T. Chung, C.Y. Lam and J. Qian, A staggered discontinuous Galerkin method for the simulation of seismic waves with surface topography. Geophysics 80 (2015) T119-T135.

[19] G. Cohen and S. Pernet, Finite Element and Discontinuous Galerkin Methods for Transient Wave Equations. Springer, Dordrecht (2017).

[20] R. Courant, K. Friedrichs and H. Lewy, Über die partiellen Differenzengleichungen der mathematischen Physik. Math. Ann. 100 (1928) 32-74.

[21] S. Delcourte and N. Glinsky, Analysis of a high-order space and time discontinuous Galerkin method for elastodynamic equations. Application to 3D wave propagation. ESAIM:M2AN 49 (2015) 1085-1126.

[22] A. Ezziani, Modélisation mathématique et numérique de la propagation d'ondes dans les milieux viscoélastiques et poroélastiques. Ph.D. thesis, Univ. Paris IX Dauphine (2005).

[23] C. Farhat, M. Lesoinne and N. Maman, Mixed explicit/implicit time integration of coupled aeroelastic problems: three field formulation, geometric conservation and distributed solution. Int. J. Numer. Meth. Fluids 21 (1995) 807-835.

[24] C.A. Felippa, K.C. Park and C. Farhat, Partitioned analysis of coupled mechanical systems. Comput. Methods Appl. Mech. Eng. 190 (2001) 3247-3270.

[25] R.W. Graves, Simulating seismic wave propagation in 3D elastic media using staggered-grid finite differences. Bull. Seismol. Soc. Amer. 86 (1996) 1091-1106.

[26] A.E. Green and P.M. Naghdi, A general theory of an elastic-plastic continuum. Arch. Rational Mech. Anal. 18 (1965) $251-281$.

[27] J.-P. Groby, Modélisation de la propagation des ondes élastiques générées par un séisme proche ou éloigné à l'intérieur d'une ville. Ph.D. thesis, Universit de la Méditerranée - Aix-Marseille II (2005).

[28] W. Han and B.D. Reddy, Plasticity. Springer, New York (1999).

[29] M. Hofacker and C. Miehe, Continuum phase field modeling of dynamic fracture: variational principles and staggered FE implementation. Int. J. Fract. 178 (2012) 113-129.

[30] M. Jirásek, Nonlocal theories in continuum mechanics. Acta Polytech. 44 (2004) 16-34.

[31] P. Joly and C. Tsogka, Finite Element Methods with Discontinuous Displacement. Chapter 11. Chapman \& Hall/CRC, Boca Raton, FL (2008).

[32] L.M. Kachanov, Time of rupture process under creep conditions. Izv. Akad. Nauk SSSR 8 (1958) 26.

[33] R. Kolman, J. Plešek, J. Červ and D. Gabriel, Grid dispersion analysis of plane square biquadratic serendipity finite elements in transient elastodynamics. Int. J. Numer. Meth. Eng. 96 (2013) 1-28.

[34] R. Kolman, J. Plešek, J. Červ, M. Okrouhlík and P. Pař́k, Temporal-spatial dispersion and stability analysis of finite element method in explicit elastodynamics. Int. J. Numer. Meth. Eng. 106 (2016) 113-128. 
[35] M. Kružík and T. Roubíček, Mathematical Methods in Continuum Mechanics of Solids. Springer, Switzeland (2019).

[36] G.A. Maugin, The saga of internal variables of state in continuum thermo-mechanics (1893-2013). Mech. Res. Commun. 69 (2015) 79-86.

[37] A. Mielke and T. Roubíček, Rate-Independent Systems - Theory and Application. Springer, New York (2015).

[38] Y.N. Rabotnov, Creep Problems in Structural Members. North-Holland, Amsterdam (1969).

[39] T. Roubíček, Nonlinear Partial Differential Equations with Applications, 2nd edition. Birkhäuser, Basel (2013).

[40] T. Roubíček, An energy-conserving time-discretisation scheme for poroelastic media with phase-field fracture emitting waves and heat. Disc. Cont. Dynam. Syst. S 10 (2017) 867-893.

[41] T. Roubíček and C.G. Panagiotopoulos, Energy-conserving time-discretisation of abstract dynamical problems with applications in continuum mechanics of solids. Numer. Funct. Anal. Optim. 38 (2017) 1143-1172.

[42] T. Roubíček and R. Vodička, A monolithic model for phase-field fracture and waves in solid-fluid media towards earthquakes. Int. J. Fract. 219 (2019) 135.

[43] T. Roubíček, M. Kružík, V. Mantič, C.G. Panagiotopoulos, R. Vodička and J. Zeman, Delamination and adhesive contacts, their mathematical modeling and numerical treatment, edited by V. Mantič, 2nd edition. In: Math. Methods and Models in Composites. Imperial College Press (2017).

[44] T. Roubíček, C.G. Panagiotopoulos, and C. Tsogka, Explicit time-discretisation of elastodynamics with some inelastic processes at small strains. Preprint arXiv: 1903.11654 (2019).

[45] G. Scarella, Etude théorique et numérique de la propagation d'ondes en présence de contact unilatéral dans un milieu fissuré. Ph.D. thesis, Univ. Paris Dauphine (2004).

[46] A. Schlüter, A. Willenbücher, C. Kuhn, and R. Müller. Phase field approximation of dynamic brittle fracture. Comput. Mech. 54 (2014) 1141-1161.

[47] S. Seifi, K.C. Park and H.S. Park, A staggered explicit-implicit finite element formulation forelectroactive polymers. Comput. Methods Appl. Mech. Eng. 337 (2018) 150-164.

[48] B. Straughan, Stability and Wave Motion in Porous Media. Springer, New York (2008).

[49] R. Temam, Mathematical Problems in Plasticity (French original in 1983). Gauthier-Villars, Paris (1985).

[50] C. Tsogka, Modelisation mathématique et numérique de la propagation des ondes élastiques tridimensionnelles dans des milieux fissurés. Ph.D. thesis, Univ. Paris IX Dauphine (1999).

[51] J. Virieux, SH-wave propagation in heterogeneous media: Velocity-stress finite-difference method. Geophysics 49 (1984) $1933-1957$. 